\title{
Probing many-body dynamics on a 51-atom quantum simulator
}

\author{
Hannes Bernien, ${ }^{1}$ Sylvain Schwartz, ${ }^{1,2}$ Alexander Keesling, ${ }^{1}$ Harry Levine, ${ }^{1}$ Ahmed Omran, ${ }^{1}$ Hannes Pichler,${ }^{3,1}$ \\ Soonwon Choi, ${ }^{1}$ Alexander S. Zibrov, ${ }^{1}$ Manuel Endres ${ }^{4}$ Markus Greiner, ${ }^{1}$ Vladan Vuletić, ${ }^{2}$ and Mikhail D. Lukin ${ }^{1}$ \\ ${ }^{1}$ Department of Physics, Harvard University, Cambridge, MA 02138, USA \\ ${ }^{2}$ Department of Physics and Research Laboratory of Electronics, \\ Massachusetts Institute of Technology, Cambridge, MA 02139, USA \\ ${ }^{3}$ ITAMP, Harvard-Smithsonian Center for Astrophysics, Cambridge, MA 02138, USA \\ ${ }^{4}$ Division of Physics, Mathematics and Astronomy, California Institute of Technology, Pasadena, CA 91125, USA
}

\begin{abstract}
Controllable, coherent many-body systems provide unique insights into fundamental properties of quantum matter, allow for the realization of novel quantum phases, and may ultimately lead to computational systems that are exponentially superior to existing classical approaches. Here, we demonstrate a novel platform for the creation of controlled many-body quantum matter. Our approach makes use of deterministically prepared, reconfigurable arrays of individually controlled, cold atoms. Strong, coherent interactions are enabled by coupling to atomic Rydberg states. We realize a programmable Ising-type quantum spin model with tunable interactions and system sizes of up to 51 qubits. Within this model we observe transitions into ordered states (Rydberg crystals) that break various discrete symmetries, verify high-fidelity preparation of ordered states, and investigate dynamics across the phase transition in large arrays of atoms. In particular, we observe a novel type of robust many-body dynamics corresponding to persistent oscillations of crystalline order after a sudden quantum quench. These observations enable new approaches for exploring many-body phenomena and open the door for realizations of novel quantum algorithms.
\end{abstract}

The realization of fully controlled, coherent many-body quantum systems is an outstanding challenge in modern science and engineering. As quantum simulators, they can provide unique insights into strongly correlated quantum systems and the role of quantum entanglement [1, and enable realizations and studies of new states of matter, even away from equilibrium. These systems also form the basis for the realization of quantum information processors [2]. While basic building blocks of such processors have been demonstrated in systems of a few coupled qubits [3-5], the current challenge is to increase the number of coherently coupled qubits to potentially perform tasks that are beyond the reach of modern classical machines.

A number of physical platforms are currently being explored to reach these challenging goals. Systems composed of about 10-20 individually controlled atomic ions have been used to create entangled states and explore quantum simulations of Ising spin models [6, 7]. Similarly sized systems of programmable superconducting qubits have been recently implemented [8, 9]. Quantum simulations have been carried out in larger systems of over 100 trapped ions without individual addressing and control [10. Strongly interacting quantum dynamics has been explored using optical lattice simulators 11. These systems are already addressing computationally difficult problems in quantum dynamics [12] and the fermionic Hubbard model [13. Larger-scale Isinglike machines have been realized in superconducting [14] and optical [15] systems but these realizations lack either coherence or quantum nonlinearity that are essential for achieving full quantum speedup.

\section{STRONGLY INTERACTING ATOM ARRAYS}

Our approach makes use of atom-by-atom assembly to deterministically prepare arrays of individually trapped cold neutral ${ }^{87} \mathrm{Rb}$ atoms in optical tweezers [16, 18, S1]. Controlled, coherent interactions between these atoms are introduced by coupling them to Rydberg states (Fig. 1 1a). This results in repulsive van der Waals interactions $\left(V_{i j}=C / R_{i j}^{6}, C>0\right)$ between Rydberg atom pairs at a distance $R_{i j}$ [19]. Such interactions have already been used for realizing quantum gates 2022 , implementing strong photon-photon interactions 23 , and studying many-body physics [24 26]. The quantum dynamics of this system is governed by the Hamiltonian

$$
\frac{\mathcal{H}}{\hbar}=\sum_{i} \frac{\Omega_{i}}{2} \sigma_{x}^{i}-\sum_{i} \Delta_{i} n_{i}+\sum_{i<j} V_{i j} n_{i} n_{j},
$$

where $\Delta_{i}$ are the detunings of the driving lasers from the Rydberg state (Fig. 1p), $\sigma_{x}^{i}=\left|g_{i}\right\rangle\left\langle r_{i}|+| r_{i}\right\rangle\left\langle g_{i}\right|$ describes the coupling between the ground state $|g\rangle$ and the Rydberg state $|r\rangle$ of an atom at position $i$, driven at Rabi frequency $\Omega_{i}$, and $n_{i}=\left|r_{i}\right\rangle\left\langle r_{i}\right|$. In general, within this platform, one can program the control parameters $\Omega_{i}, \Delta_{i}$ by changing laser intensities and detunings in time. Here, we focus on homogeneous coherent coupling $\left(\left|\Omega_{i}\right|=\Omega, \Delta_{i}=\Delta\right)$. The interaction strength $V_{i j}$ is tuned by either varying the distance between the atoms or coupling them to a different Rydberg state.

The experimental protocol that we implement is depicted in Fig. 17. First, atoms are loaded from a magneto-optical trap into a tweezer array created by an acousto-optic deflector (AOD). We then use a measurement and feedback procedure that eliminates the entropy 


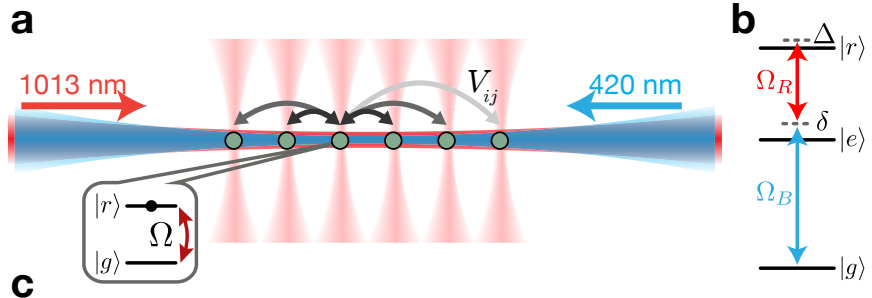

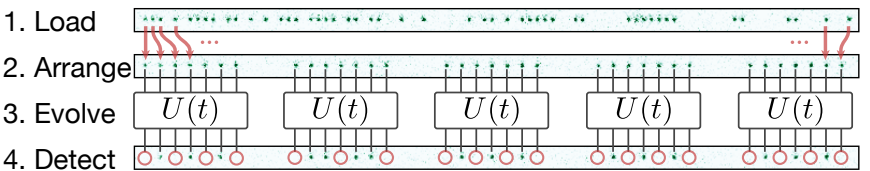

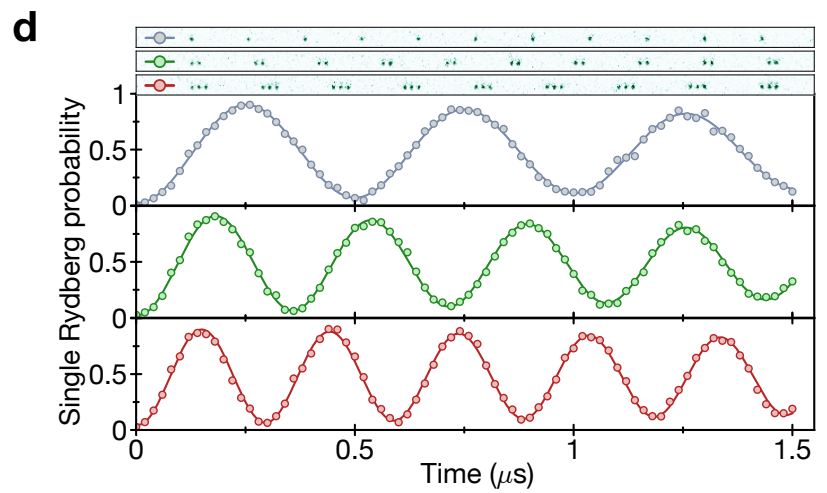

FIG. 1: Experimental platform. a, Individual ${ }^{87} \mathrm{Rb}$ atoms are trapped using optical tweezers and arranged into defectfree arrays. Coherent interactions $V_{i j}$ between the atoms are enabled by exciting them to a Rydberg state, with strength $\Omega$ and detuning $\Delta$. $\mathbf{b}$, A two photon process is used to couple the ground state $|g\rangle=\left|5 S_{1 / 2}, F=2, m_{F}=-2\right\rangle$ to the Rydberg state $|r\rangle=\left|71 S_{1 / 2}, J=1 / 2, m_{J}=-1 / 2\right\rangle$ via an intermediate state $|e\rangle=\left|6 P_{3 / 2}, F=3, m_{F}=-3\right\rangle$ using circularly polarized $420 \mathrm{~nm}$ and $1013 \mathrm{~nm}$ lasers (typically $\delta \sim 2 \pi \times 560 \mathrm{MHz} \gg$ $\Omega_{B}, \Omega_{R} \sim 2 \pi \times 60,36 \mathrm{MHz}$ ). c, The experimental protocol consists of loading the atoms into a tweezer array (1) and rearranging them into a preprogrammed configuration (2). After this, the system evolves under $U(t)$ with tunable parameters $\Delta(t), \Omega(t), V_{i j}$. This can be implemented in parallel on several non-interacting sub-systems (3). We then detect the final state by fluorescence imaging (4). d, For resonant driving $(\Delta=0)$, isolated atoms (blue points) display Rabi oscillations between $|g\rangle$ and $|r\rangle$. Arranging the atoms into fully blockaded clusters of $N=2$ (green) and $N=3$ (red) atoms results in only one excitation being shared between the atoms in the cluster, while the Rabi frequency is enhanced by $\sqrt{N}$. The probability to detect more than one excitation in the cluster is $\leq 5 \%$. Error bars indicate $68 \%$ confidence intervals (CI) and are smaller than the marker size.

associated with the probabilistic trap loading and results in the rapid production of defect-free arrays with over 50 laser cooled atoms as described previously [S1. These atoms are prepared in a preprogrammed spatial configuration in a well-defined internal ground state $|g\rangle$ (Supplementary Information). We then turn off the traps and let the system evolve under the unitary time evolution
$U(\Omega, \Delta, t)$, which is realized by coupling the atoms to the Rydberg state $|r\rangle=\left|71 S_{1 / 2}\right\rangle$ with laser light along the array axis (Fig. 17). The final states of individual atoms are detected by turning the traps back on, and imaging the recaptured ground state atoms via atomic fluorescence, while the anti-trapped Rydberg atoms are ejected (Supplementary Information).

The strong, coherent interactions between Rydberg atoms provide an effective coherent constraint that prevents simultaneous excitation of nearby atoms into Rydberg states. This is the essence of the so-called Rydberg blockade [19], demonstrated in Fig. 11. When two atoms are sufficiently close so that their Rydberg-Rydberg interactions $V_{i j}$ exceed the effective Rabi frequency $\Omega$, then multiple Rydberg excitations are suppressed. This defines the Rydberg blockade radius, $R_{b}$, for which $V_{i j}=\Omega$ $\left(R_{b}=9 \mu \mathrm{m}\right.$ for $|r\rangle=\left|71 S_{1 / 2}\right\rangle$ and $\Omega=2 \pi \times 2 \mathrm{MHz}$ as used here). In the case of resonant driving of atoms separated by $a=24 \mu \mathrm{m}$, we observe Rabi oscillations associated with non-interacting atoms (blue curve on Fig. 1 d). However, the dynamics change significantly as we bring multiple atoms close to each other $\left(a=2.95 \mu \mathrm{m}<R_{b}\right)$. In this case, we observe Rabi oscillations between the ground state and a collective $\mathrm{W}$-state with exactly one excitation $\sim \sum_{i} \Omega_{i}\left|g_{1} \ldots r_{i} \ldots g_{N}\right\rangle$ with the characteristic $\sqrt{N}$-scaling of the collective Rabi frequency [25, 27, 28]. These observations allow us to quantify the coherence properties of our system (see Supplementary Information for details). In particular, the contrast of Rabi oscillations in Fig. $1 \mathrm{~d}$ is mostly limited by the state detection fidelity (93\% for $|r\rangle$ and $\sim 98 \%$ for $|g\rangle$, Supplementary Information). The individual Rabi frequencies are controlled to better than $3 \%$ across the array, while the coherence time is ultimately limited by the small probability of spontaneous emission from the intermediate state $|e\rangle$ during the laser pulse (scattering rate $0.022 / \mu \mathrm{s}$, Supplementary Information).

\section{PROGRAMMABLE QUANTUM SIMULATOR}

In the case of homogeneous coherent coupling considered here, Hamiltonian (1) closely resembles the paradigmatic Ising model for effective spin- $1 / 2$ particles with variable interaction range. Its ground state exhibits a rich variety of many-body phases that break distinct spatial symmetries (Fig. 2a). Specifically, at large, negative values of $\Delta / \Omega$, its ground state corresponds to all atoms in the state $|g\rangle$, corresponding to paramagnetic or disordered phase. As $\Delta / \Omega$ is increased towards large positive values, the number of atoms in $|r\rangle$ rises and interactions between them become significant. This gives rise to spatially ordered phases where Rydberg atoms are regularly arranged across the array, resulting in 'Rydberg crystals' with different spatial symmetries [29 31, as illustrated in Fig. 2a. The origin of these correlated states 

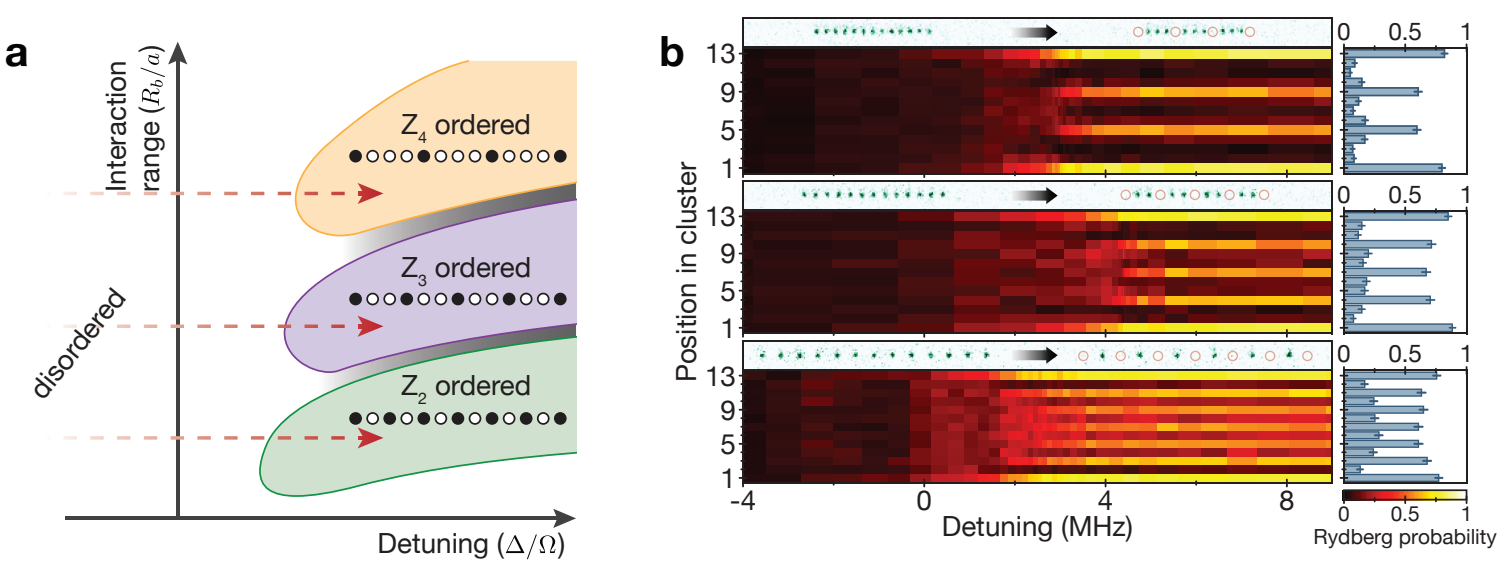

FIG. 2: Phase diagram and buildup of crystalline phases. a, The schematic ground-state phase diagram of Hamiltonian (1) displays phases with various broken symmetries depending on the interaction range $R_{b} / a$ ( $R_{b}$ blockade radius, $a$ trap spacing) and detuning $\Delta$ (see main text). Shaded areas indicate potential incommensurate phases (diagram adapted from [29]). b, The buildup of Rydberg crystals on a 13 atom array is observed by slowly changing the laser parameters as indicated by the red arrows in a (see also Fig. 3a). The bottom panel shows a configuration where the atoms are $a=5.9 \mu \mathrm{m}$ apart which results in a nearest neighbor interaction of $V_{i, i+1}=2 \pi \times 24 \mathrm{MHz}$ and leads to a $\mathrm{Z}_{2}$ order where every other atom is excited to the Rydberg state $|r\rangle$. The right bar plot displays the final, position-dependent Rydberg probability (error bars denote $68 \% \mathrm{CI})$. The configuration in the middle panel $\left(a=3.67 \mu \mathrm{m}, V_{i, i+1}=2 \pi \times 414.3 \mathrm{MHz}\right)$ results in $\mathrm{Z}_{3}$ order and the top panel $\left(a=2.95 \mu \mathrm{m}, V_{i, i+1}=2 \pi \times 1536 \mathrm{MHz}\right)$ in a $\mathrm{Z}_{4}$ ordered phase. For each configuration, we show a single-shot fluorescence image before (left) and after (right) the pulse. Red circles highlight missing atoms, which are attributed to Rydberg excitations.

can be understood intuitively by first considering the situation when $V_{i, i+1} \gg \Delta \gg \Omega \gg V_{i, i+2}$, i.e. blockade for neighboring atoms but negligible interaction between next-nearest neighbors. In this case, the ground state corresponds to a Rydberg crystal breaking $\mathrm{Z}_{2}$ translational symmetry that is analogous to antiferromagnetic order in magnetic systems. Moreover, by tuning the parameters such that $V_{i, i+1}, V_{i, i+2} \gg \Delta \gg \Omega \gg V_{i, i+3}$ and $V_{i, i+1}, V_{i, i+2}, V_{i, i+3} \gg \Delta \gg \Omega \gg V_{i, i+4}$, we obtain arrays with broken $\mathrm{Z}_{3}$ and $\mathrm{Z}_{4}$ symmetries, respectively (Fig. 2).

To prepare the system in these phases, we dynamically control the detuning $\Delta(t)$ of the driving lasers to adiabatically transform the ground state of the Hamiltonian from a product state of all atoms in $|g\rangle$ into crystalline states [31, 32]. In the experiment, we first prepare all atoms in state $|g\rangle=\left|5 S_{1 / 2}, F=2, m_{F}=-2\right\rangle$ by optical pumping. We then switch on the laser fields and sweep the two-photon detuning from negative to positive values using a functional form shown in Fig. 3a. Fig. 20 displays the resulting single atom trajectories in a group of 13 atoms for three different interaction strengths as we vary the detuning $\Delta$. In each of these instances, we observe a clear transition from the initial state $\left|g_{1}, \ldots, g_{13}\right\rangle$ to an ordered state of different broken symmetry. The distance between the atoms determines the interaction strength which leads to different crystalline orders for a given final detuning. To achieve a $\mathrm{Z}_{2}$ order, we arrange the atoms with a spacing of $5.9 \mu \mathrm{m}$, which results in a nearest neighbor interaction of $V_{i, i+1}=2 \pi \times 24 \mathrm{MHz} \gg \Omega=2 \pi \times 2 \mathrm{MHz}$, while the next-nearest neighbor interaction is small $(2 \pi \times 0.38 \mathrm{MHz})$. This results in a buildup of antiferromagnetic order where every other trap site is occupied by a Rydberg atom $\left(\mathrm{Z}_{2}\right.$ order). By reducing the spacing between the atoms to $3.67 \mu \mathrm{m}$ and $2.95 \mu \mathrm{m}, \mathrm{Z}_{3^{-}}$and $\mathrm{Z}_{4^{-}}$ orders are respectively observed (Fig. $2 \mathrm{~b}$ ).

We benchmark the performance of the quantum simulator by comparing the measured $\mathrm{Z}_{2}$ order buildup with theoretical predictions for a $N=7$ atom system, obtained via exact numerical simulations. As shown in Fig. 3, this fully coherent simulation without free parameters yields excellent agreement with the observed data when the finite detection fidelity is accounted for. The evolution of the many-body states in Fig. 3r shows that we measure the perfect antiferromagnetic state with $54(4) \%$ probability. When corrected for the known detection infidelity, we find that the desired many-body state is reached with a probability of $p=77(6) \%$.

To investigate how the preparation fidelity depends on system size, we perform detuning sweeps on arrays of various sizes (Fig. 4 4 ). We find that the probability of observing the system in the many-body ground state at the end of the sweep decreases as the the system size is increased. However, even at system sizes as large as 51 atoms, the perfectly ordered crystalline many-body state is obtained with $p=0.11(2) \%(p=0.9(2) \%$ when corrected for detection fidelity), which is remarkable in view of the exponentially large $2^{51}$-dimensional Hilbert space of the system. Furthermore, we find that this state 


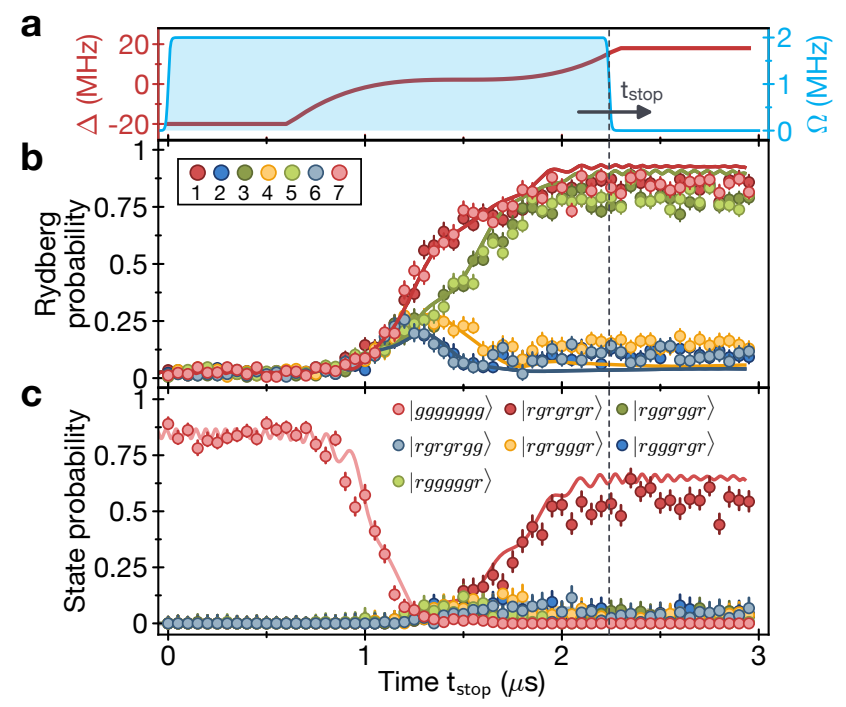

FIG. 3: Comparison with a fully coherent simulation. a, The laser driving consists of a square shaped pulse $\Omega(t)$ with a detuning $\Delta(t)$ that is chirped from negative to positive values. $\mathbf{b}$, Time evolution of Rydberg excitation probability for each atom in a $N=7$ atom cluster (colored points), obtained by varying the duration of laser excitation pulse $\Omega(t)$. The corresponding curves are theoretical single atom trajectories obtained by an exact simulation of quantum dynamics with (1), the functional form of $\Delta(t)$ and $\Omega(t)$ used in the experiment, and finite detection fidelity. c, Evolution of the seven most probable many-body states. The target state is reached with $54(4) \%$ probability $(77(6) \%$ when corrected for finite detection fidelity). Error bars denote $68 \%$ CI.

with perfect $\mathrm{Z}_{2}$ order is by far the most commonly observed many-body state (Fig. $4 \mathrm{~b}$ ).

\section{QUANTUM DYNAMICS ACROSS A PHASE TRANSITION}

We next present a detailed study of the transition into the $\mathrm{Z}_{2}$ phase in an array of 51 atoms (Fig. 5). In single instances of the experiment we observe long ordered chains where the atomic states alternate between Rydberg and ground state. These ordered domains can be separated by domain walls that consist of two neighboring atoms in the same electronic state (Fig. 5a) 33 .

The domain wall density can be used to quantify the transition from the disordered phase into the ordered $\mathrm{Z}_{2}$ phase as a function of detuning $\Delta$. As the system enters the $\mathrm{Z}_{2}$ phase, ordered domains grow in size, leading to a substantial reduction in the domain wall density (blue points in Fig. 5p). Consistent with expectations for an Ising-type second-order quantum phase transition [33, we observe domains of fluctuating lengths close to the transition point between the two phases, which is reflected by a pronounced peak in the variance of the den-
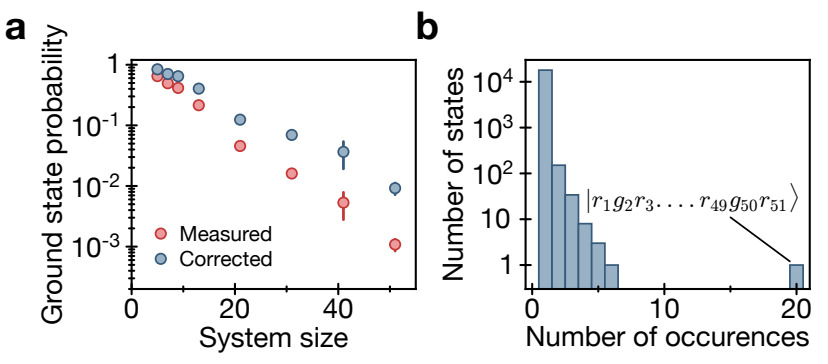

FIG. 4: Scaling behavior. a, Preparation fidelity of the crystalline ground state as a function of cluster size. The red dots are the measured values and the blue dots are corrected for finite detection fidelity (Supplementary Information). Error bars denote $68 \%$ CI. b, Number of observed many-body states per number of occurrences out of 18439 experimental realizations in a 51-atom cluster. The most occurring state is the ground state of the many-body Hamiltonian.

sity of domain walls. Consistent with predictions from finite-size scaling analysis 29, 34, this peak is shifted towards positive values of $\Delta / \Omega$. The measured position of the peak is $\Delta \simeq 0.5 \Omega$. The observed domain wall density is in excellent agreement with fully coherent simulations of the quantum dynamics based on 51-atom matrix product states (blue line); however, these simulations underestimate the variance at the phase transition (Supplementary Information).

At the end of the sweep, deep in the $Z_{2}$ phase $(\Delta / \Omega \gg$ 1 ) we can neglect $\Omega$ such that the Hamiltonian $(1)$ becomes essentially classical. In this regime, the measured domain wall number distribution allows us to directly infer the statistics of excitations created when crossing the phase transition. From 18439 experimental realizations we obtain the distribution depicted in Fig. 5. with an average of 9.01(2) domain walls. From a maximum-likelihood estimation we obtain the distribution corrected for detection fidelity (Supplementary Information), which corresponds to a state that has on average 5.4 domain walls. These domain walls are most likely created due to non-adiabatic transitions from the ground state when crossing the phase transition, where the energy gap becomes minimal [35. In addition, the preparation fidelity is also limited by spontaneous emission during the laser pulse (an average number of 1.1 photons is scattered per $\mu$ s for the entire array, see Supplementary Information).

To further characterize the created $\mathrm{Z}_{2}$ ordered state, we evaluate the correlation function

$$
g_{i j}^{(2)}=\left\langle n_{i} n_{j}\right\rangle-\left\langle n_{i}\right\rangle\left\langle n_{j}\right\rangle
$$

where the average $\langle\cdots\rangle$ is taken over experimental repetitions. We find that the correlations decay exponentially over distance with a decay length of $\xi=3.03(6)$ sites (see Fig. 5d and Supplementary Information). 
a
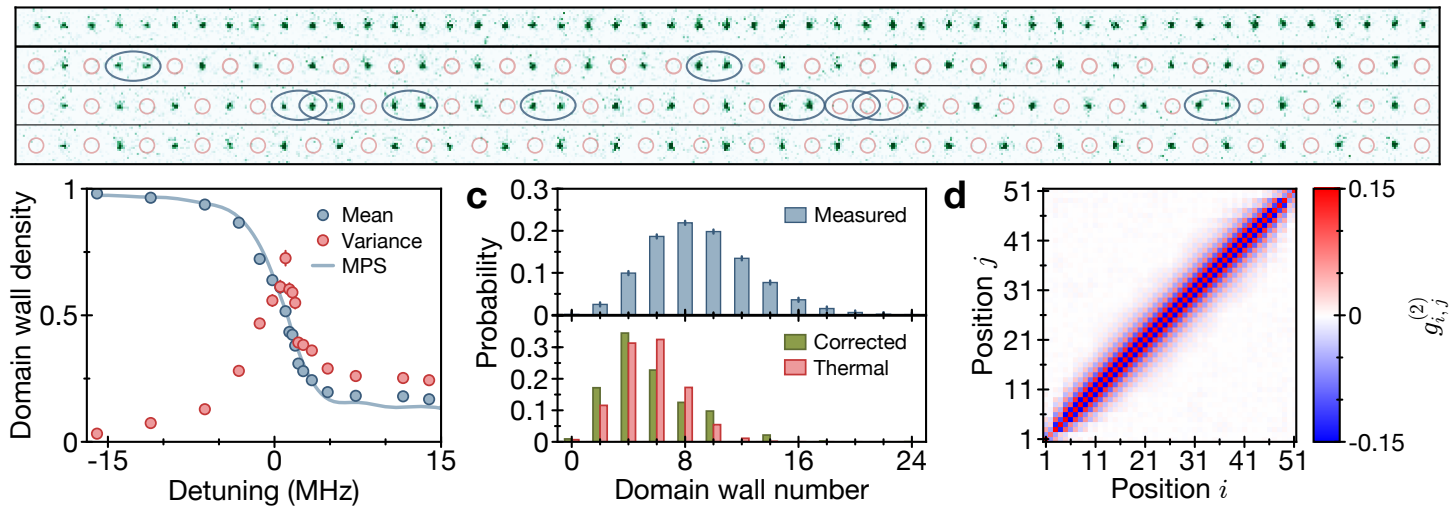

FIG. 5: Quantifying $\mathbf{Z}_{2}$ order in a $N=51$ atom array. a, Single-shot fluorescence images of a 51 atom array before applying the adiabatic pulse (top row) and after the pulse (bottom three rows correspond to three separate instances). Red circles mark missing atoms, which are attributed to Rydberg excitations. Domain walls are identified as either two neighboring atoms in the same state or a ground state atom at the edge of the array (Supplementary Information), and are indicated with ellipses. Long $\mathrm{Z}_{2}$ ordered chains between domain walls can be observed. $\mathbf{b}$, Blue points show the mean of the domain wall density as a function of detuning during the sweep. Error bars show the standard error of the mean, and are smaller than the marker size. The red points are the corresponding variances, where the error bars represent one standard deviation. The onset of the phase transition is witnessed by a decrease in the domain wall density and a peak in the variance (see main text for details). Each point is obtained from $\sim 1000$ realizations. The solid blue curve is a fully coherent MPS simulation without free parameters (bond dimension $D=256$ ), taking measurement fidelities into account. c, Domain wall number distribution for $\Delta=2 \pi \times 14 \mathrm{MHz}$, obtained from 18439 experimental realizations (blue bars, top plot). Error bars indicate $68 \%$ CI. Owing to the boundary conditions, only even numbers of domain walls can appear (Supplementary Information). Green bars in the bottom plot show the distribution obtained by correcting for finite detection fidelity using a maximum likelihood method (Supplementary Information), which results in an average number of 5.4 domain walls. Red bars show the distribution of a thermal state with the same mean domain wall density (Supplementary Information). d, Measured correlation function (2) in the $\mathrm{Z}_{2}$ phase.

Finally, Fig.6]demonstrates that our approach also enables the study of coherent dynamics of many-body systems far from equilibrium. Specifically, we focus on the quench dynamics of Rydberg crystals initially prepared deep in the $\mathrm{Z}_{2}$ ordered phase, as we suddenly change the detuning $\Delta(t)$ to the single-atom resonance $\Delta=0$ (Fig. 6a). After such a quench, we observe oscillations of many-body states between the initial crystal and a complementary crystal where each internal atomic state is inverted (Fig. 6a). We find that these oscillations are remarkably robust, persisting over several periods with a frequency that is largely independent of the system size. This is confirmed by measuring the dynamics of the domain wall density, signaling the appearance and disappearance of the crystalline states, shown in Fig. 6 $\mathrm{p}$ for arrays of 9 and 51 atoms. We find that the initial crystal repeatedly revives with a period that is slower by a factor $\sim 1.4$ compared to the Rabi oscillation period for independent, non-interacting atoms.

\section{DISCUSSION}

Several important features of these experimental observations should be noted. First of all, our $\mathrm{Z}_{2}$ ordered state cannot be characterized by a simple thermal ensemble. More specifically, if an effective temperature is estimated based on the measured domain wall density, the corresponding thermal ensemble predicts a correlation length $\xi_{\text {th }}=4.48(3)$, which is significantly longer than the measured value $\xi=3.03(6)$. Such a discrepancy is also reflected in distinct probability distributions for the number of domain walls (Fig. 5c). These observations suggest that the system does not thermalize within the timescale of the $\mathrm{Z}_{2}$ state preparation.

Even more striking is the coherent and persistent oscillation of the crystalline order after the quantum quench. With respect to the quenched Hamiltonian $(\Delta=0)$, the energy density of our $\mathrm{Z}_{2}$ ordered state corresponds to that of an infinite-temperature ensemble within the manifold constrained by Rydberg blockade. Also, our Hamiltonian does not have any explicit conserved quantities other than total energy. Nevertheless, the oscillations persist well beyond the natural timescale of local relaxation $\sim 1 / \Omega$ as well as the fastest timescale, $1 / V_{i, i+1}$.

To understand these observations, we consider a simplified model where the effect of long-range interactions is neglected, and nearest-neighbor interactions are replaced by hard constraints on neighboring excitations of Rydberg states [29]. In this limit, the qualitative behav- 
a

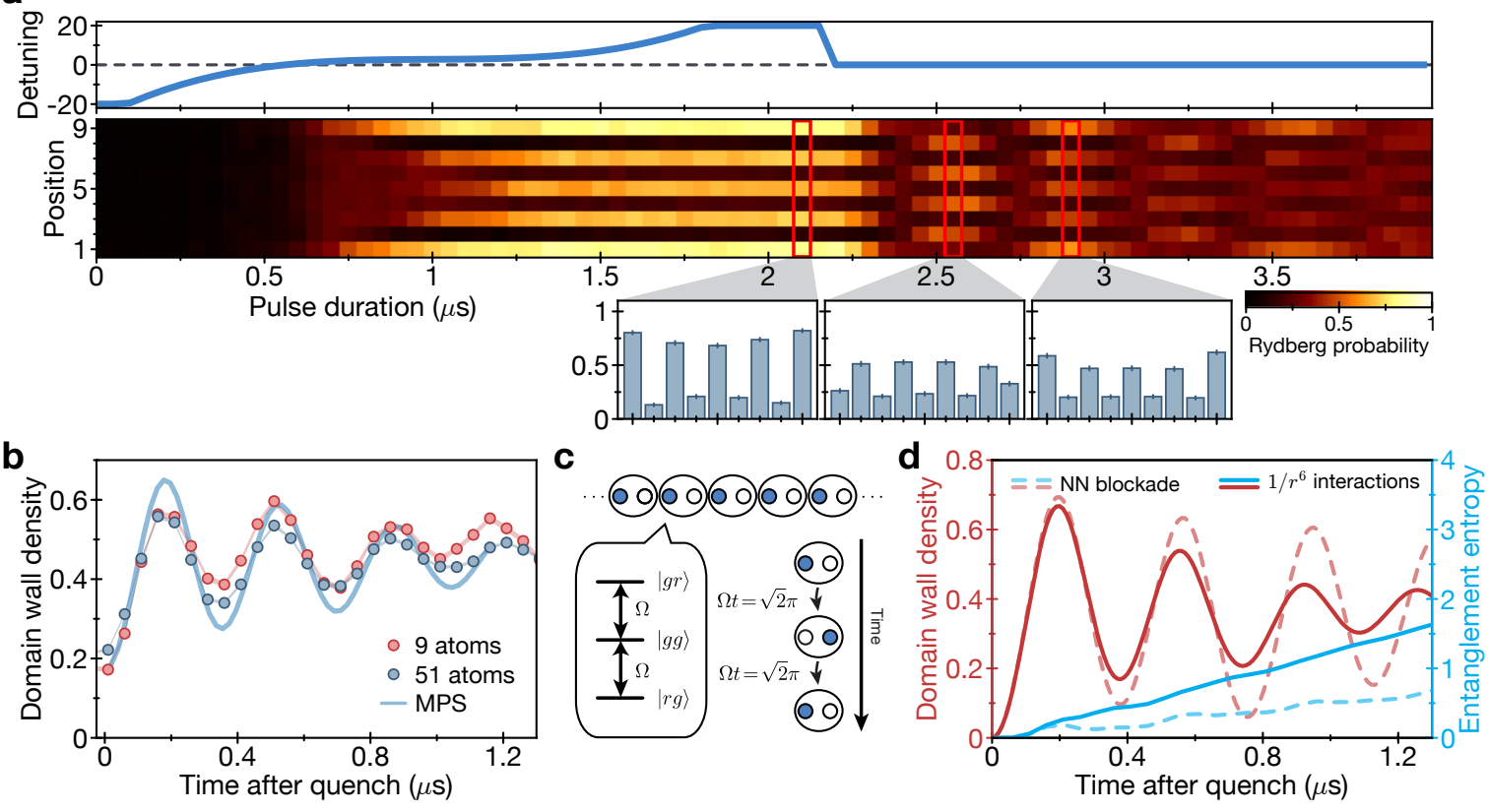

FIG. 6: Emergent oscillations in many-body dynamics. a, Schematic sequence (top, showing $\Delta(t)$ ) involves adiabatic preparation and then a sudden quench to single-atom resonance. The heat map shows the single atom trajectories for a 9 atom cluster. We observe that the initial (left inset) crystal with a Rydberg excitation at every odd trap site collapses after the quench and a crystal with an excitation at every even site builds up (middle inset). At a later time the initial crystal revives (right inset). Error bars denote $68 \%$ CI. b, Density of domain walls after the quench. The dynamics decay slowly on a timescale of $0.88 \mu \mathrm{s}$. Shaded region represents the standard error of the mean. Solid blue line is a fully coherent MPS simulation 36 . with bond dimension $D=256$, taking into account measurement fidelity. c, Toy model of non-interacting dimers (see main text). d, Numerical calculations of the dynamics after a quench starting from an ideal 25 atom crystal, obtained from exact diagonalization. Domain wall density as a function of time (red), and growth of entanglement entropy of the half chain (13 atoms) (blue). Dashed lines take into account only nearest neighbor blockade constraint. Solid lines correspond to the full $1 / R^{6}$ interaction potential.

ior of the quench dynamics can be understood in terms of dimerized spins (Fig. 6c); owing to the blockade constraint, each dimer forms an effective spin-1 system with three states $|r g\rangle,|g g\rangle$, and $|g r\rangle$, where the resonant drive "rotates" the three states over the period $\sqrt{2}(2 \pi / \Omega)$, close to that observed experimentally. While this qualitative picture does not take into account the strong interactions (constraints) between neighboring dimers, it can be extended by considering a minimal variational ansatz for the many-body wave function based on matrix product state (MPS) that respects all blockade constraints (Supplementary Information). Using the time-dependent variational principle, we derive analytical equations of motion and obtain a crystalline-order oscillation with frequency $\sim \Omega / 1.51$, which is within $10 \%$ of the experimental observations. These considerations are supported by various numerical simulations. Indeed, the exact numerics predict that this simplified model exhibits crystal oscillations with the observed frequency, while the entanglement entropy grows at a rate much smaller than $\Omega$, indicating that the oscillation persists over many cycles (Fig. 6d and Supplementary Information). The ad- dition of long-range interactions leads to a faster decay of the oscillations, with a timescale that is determined by $\sim 1 / V_{i, i+2}$, in good agreement with experimental observations (Fig. 6p), while the entanglement entropy also grows on this time scale (Fig. 6d).

Thus, our observations and analysis indicate that the decay of crystal oscillation is governed by weak nextnearest-neighbor interactions. This relatively slow thermalization is rather unexpected, since our Hamiltonian, with or without long-range interactions, is far from any known integrable systems [29, and features neither strong disorder [37] nor explicitly conserved quantities [38. Instead, our observations are associated with constrained dynamics due to Rydberg blockade and large separations of timescales $V_{i, i+1} \gg \Omega \gg V_{i, i+2}$ [S8], which gives rise to so-called quantum dimer models, with the Hilbert space dimension determined by the golden ratio $\sim(1+\sqrt{5})^{N} / 2^{N}$, that are known to possess non-trivial dynamics [40, 41]. While these considerations provide important insights into the origin of robust emergent dynamics, we emphasize that our results challenge conventional theoretical concepts and warrant further studies. 
For example, the connections to recent work on dynamics of Fibonacci anyons in constrained Hilbert spaces [42, 43. and on many-body localization in translation-invariant systems 44 should be explored.

\section{OUTLOOK}

Our observations demonstrate that Rydberg excitation of neutral atom arrays constitutes an exceptionally promising platform for studying quantum dynamics and quantum simulations in large systems. Our method can be extended and improved in a number of ways. Coherence properties of atoms can be improved by increasing intermediate state detuning to further suppress spontaneous emission and by Raman sideband cooling the atomic motion to the ground state to eliminate the residual Doppler shifts. Individual qubit rotations around the $z$-axis can be implemented using light shifts associated with trap light, while a second AOD can be used for individual control of coherent rotations around other directions. Further improvement in coherence and controllability can be obtained by encoding qubits into hyperfine sublevels of the electronic ground state and using state-selective Rydberg excitation [26]. Implementing two-dimensional (2d) arrays could provide a path towards realizing thousands of traps. Such $2 \mathrm{~d}$ configurations could be realized by directly using a $2 \mathrm{~d}-\mathrm{AOD}$ or by creating a static $2 \mathrm{~d}$ lattice of traps and sorting atoms with an independent AOD, as demonstrated recently [16]. With increased loading efficiencies [45], the robust creation and control of arrays composed of hundreds of atoms is feasible.

While our current observations already allow us to gain unprecedented insights into the physics associated with transitions into ordered phases and to explore novel many-body phenomena in quantum dynamics, they can be directly extended along several directions [46]. These include studies of entanglement in large arrays [47, 48, and the generation of many-particle quantum superposition states, investigation of quantum critical dynamics and tests of the quantum Kibble-Zurek hypothesis 35, and the exploration of stable non-equilibrium phases of matter [37, 49]. Further extension may allow for studies of the interplay between long-range interactions and disorder [50], quantum scrambling [51], topological states in spin systems [42, 43] and investigation of chiral clock models associated with transitions into exotic $\mathrm{Z}_{3}$ and $\mathrm{Z}_{4}$ states 52. Finally, we note that our approach is exceptionally well suited for the realization and testing of quantum optimization algorithms with systems that are well beyond the reach of modern classical machines 53, 54. The latter may have broad potential applications ranging from modeling and optimization of chemical reactions [55] to quantum machine learning [56].

\section{ACKNOWLEGEMENTS}

We thank A. Chandran, E. Demler, M. Fleischhauer, A. Harrow, S. Hofferberth, M. Knap, T. Pohl, S. Sachdev, P. Silvi, A. Vishwanath and P. Zoller for insightful discussions. This work was supported by NSF, CUA, ARO, MURI, AFOSR, and Vannevar Bush Faculty Fellowship. H.B. acknowledges support by a Rubicon Grant of the Netherlands Organization for Scientific Research (NWO). A.O. acknowledges support by a research fellowship from the German Research Foundation (DFG). S.S. acknowledges funding from the European Union under the Marie Skłodowska Curie Individual Fellowship Programme H2020-MSCA-IF-2014 (project number 658253). H.P. acknowledges support by the National Science Foundation (NSF) through a grant at the Institute of Theoretical Atomic Molecular and Optical Physics (ITAMP) at Harvard University and the Smithsonian Astrophysical Observatory.

\section{AUTHOR CONTRIBUTIONS}

The experiments and data analysis were carried out by H.B., S.S., A.K., H.L., A.O., A.S.Z., and M.E. Theoretical analysis was performed by H.P. and S.C. All work was supervised by M.G., V.V. and M.D.L.. All authors discussed the results and contributed to the manuscript.

[1] I. Bloch, J. Dalibard, and S. Nascimbène, Nat. Phys. 8, 267 (2012).

[2] T. D. Ladd et al., Nature 464, 45 (2010).

[3] C. Monroe and J. Kim, Science 339, 1164 (2013).

[4] M. H. Devoret and R. J. Schoelkopf, Science 339, 1169 (2013).

[5] D. D. Awschalom, L. C. Bassett, A. S. Dzurak, E. L. Hu, and J. R. Petta, Science 339, 1174 (2013).

[6] T. Monz et al., Phys. Rev. Lett. 106, 130506 (2011).

[7] R. Islam et al., Science 340, 583 (2013).

[8] N. Ofek et al., Nature 536, 441 (2016).

[9] C. Song et al., arXiv:1703.10302 (2017).

[10] M. Gärttner et al., Nat. Phys. , Advanced online publication (2017).

[11] S. Kuhr, Natl. Sci. Rev. 3, 170 (2016).

[12] S. Trotzky et al., Nat. Phys. 8, 325 (2012).

[13] A. Mazurenko et al., Nature 545, 462 (2017).

[14] T. F. Rønnow et al., Science 345, 420 (2014).

[15] P. L. McMahon et al., Science 354, 614 (2016).

[16] D. Barredo, S. de Léséleuc, V. Lienhard, T. Lahaye, and A. Browaeys, Science 354, 1021 (2016).

[17] M. Endres et al., Science 354, 1024 (2016).

[18] H. Kim et al., Nat. Commun. 7, 13317 (2016).

[19] D. Jaksch et al., Phys. Rev. Lett. 85, 2208 (2000).

[20] T. Wilk et al., Phys. Rev. Lett. 104, 010502 (2010).

[21] L. Isenhower et al., Phys. Rev. Lett. 104, 010503 (2010). 
[22] M. Saffman, T. G. Walker, and K. Mølmer, Rev. Mod. Phys. 82, 2313 (2010).

[23] J. D. Pritchard et al., Phys. Rev. Lett. 105, 193603 (2010).

[24] P. Schauß et al., Nature 491, 87 (2012).

[25] H. Labuhn et al., Nature 534, 667 (2016).

[26] J. Zeiher et al., arXiv:1705.08372 (2017).

[27] Y. O. Dudin, L. Li, F. Bariani, and A. Kuzmich, Nat. Phys. 8, 790 (2012).

[28] J. Zeiher et al., Phys. Rev. X 5, 031015 (2015).

[29] P. Fendley, K. Sengupta, and S. Sachdev, Phys. Rev. B 69, 075106 (2004).

[30] H. Weimer and H. P. Büchler, Phys. Rev. Lett. 105, 230403 (2010).

[31] T. Pohl, E. Demler, and M. D. Lukin, Phys. Rev. Lett. 104, 043002 (2010).

[32] P. Schauß et al., Science 347, 1455 (2015).

[33] S. Sachdev, Quantum Phase Transitions, 2nd ed. (Cambridge University Press, 2009).

[34] S. Sachdev, K. Sengupta, and S. M. Girvin, Phys. Rev. B 66, 075128 (2002).

[35] W. H. Zurek, U. Dorner, and P. Zoller, Phys. Rev. Lett. 95, 105701 (2005).

[36] U. Schollwöck, Ann. Phys. 326, 96 (2011).

[37] M. Schreiber et al., Science 349, 842 (2015).

[38] L. D'Alessio, Y. Kafri, A. Polkovnikov, and M. Rigol, Adv. Phys. 65, 239 (2016).

[39] D. Abanin, W. De Roeck, W. W. Ho, and F. Huveneers, Comm. Math. Phys. 354, 809 (2017).

[40] I. Lesanovsky and H. Katsura, Phys. Rev. A 86, 041601 (2012).

[41] R. Moessner and K. S. Raman, Quantum dimer models, in Introduction to Frustrated Magnetism Vol. 164, pp. 437-479, Springer Berlin Heidelberg, 2011.

[42] A. Chandran, M. D. Schulz, and F. J. Burnell, Phys. Rev. B 94, 235122 (2016).

[43] A. Feiguin et al., Phys. Rev. Lett. 98, 160409 (2007).

[44] M. Schiulaz, A. Silva, and M. Müller, Phys. Rev. B 91, $184202(2015)$.

[45] B. J. Lester, N. Luick, A. M. Kaufman, C. M. Reynolds, and C. A. Regal, Phys. Rev. Lett. 115, 073003 (2015).

[46] H. Weimer, M. Müller, I. Lesanovsky, P. Zoller, and H. P. Büchler, Nat. Phys. 6, 382 (2010).

[47] P. Hauke, M. Heyl, L. Tagliacozzo, and P. Zoller, Nat. Phys. 12, 778 (2016).

[48] H. Pichler, G. Zhu, A. Seif, P. Zoller, and M. Hafezi, Phys. Rev. X 6, 041033 (2016).

[49] V. Khemani, A. Lazarides, R. Moessner, and S. L. Sondhi, Phys. Rev. Lett. 116, 250401 (2016).

[50] N. Y. Yao et al., Phys. Rev. Lett. 113, 243002 (2014).

[51] B. Swingle, G. Bentsen, M. Schleier-Smith, and P. Hayden, Phys. Rev. A 94, 040302(R) (2016).

[52] D. A. Huse and M. E. Fisher, Phys. Rev. B 29, 239 (1984).

[53] W. Lechner, P. Hauke, and P. Zoller, Sci. Adv. 1, e1500838 (2015).

[54] E. Farhi and A. W. Harrow, arXiv:1602.07674 (2016).

[55] R. Babbush, P. J. Love, and A. Aspuru-Guzik, Sci. Rep. 4, 6603 (2014).

[56] S. Lloyd, M. Mohseni, and P. Rebentrost, arXiv:1307.0411 (2013). 


\section{SUPPLEMENTARY MATERIAL}

\section{EXPERIMENTAL SETUP}

\subsection{Trapping setup and experimental sequence}

Our setup consists of a linear array of up to 101 evenly spaced optical tweezers. The tweezers are generated by feeding a multi-tone RF signal into an acousto-optic deflector (AA Opto-Electronic model DTSX-400-800.850), generating multiple deflections in the first diffraction order, and focusing them into the vacuum chamber using a $0.5 \mathrm{NA}$ objective (Mitutoyo G Plan Apo 50X). The beams have a wavelength of $808 \mathrm{~nm}$ and a waist of $\sim 0.9 \mu \mathrm{m}$.

A diagram of the experimental sequence is shown in Figure S1a. The traps are loaded from a magneto-optical trap (MOT), leading to individual tweezer single-atom loading probabilities of $\sim 0.6$. A fluorescence image of the array is taken, and the empty traps are turned off, while the filled traps are rearranged to bring the atoms into their preprogrammed positions [S1]. Following the rearrangement procedure, another image of the array is taken to preselect on instances in which the initial configuration is defect-free. After taking the second image, we optically pump all atoms into the $\left|F=2, m_{F}=-2\right\rangle$ state using a $\sigma^{-}$-polarized beam resonant to the $\left|5 S_{1 / 2}, F=2\right\rangle \rightarrow\left|5 P_{3 / 2}, F=2\right\rangle$ transition. We then turn off the traps, pulse the Rydberg lasers on a timescale of a few microseconds, and then turn the traps back on to recapture the atoms that are in the ground state $|g\rangle$ while pushing away the atoms in the Rydberg state $|r\rangle$, and finally take a third image. Because of their long lifetime, most of the Rydberg atoms escape from the trapping region before they decay back to the ground state. This provides a convenient way to detect them as missing atoms on the third image (with finite detection fidelity discussed in section 1.3). The entire experimental sequence, from MOT formation to the third image, takes $\sim 250 \mathrm{~ms}$.

\subsection{Rydberg lasers setup}

To introduce interactions within the array, we couple the atomic ground state $|g\rangle=\left|5 S_{1 / 2}, F=2, m_{F}=-2\right\rangle$ to a target Rydberg state $|r\rangle=\left|71 S_{1 / 2}, m_{J}=-1 / 2\right\rangle$. The van der Waals interaction between two ${ }^{87} \mathrm{Rb} 71 S$ atoms follows a $1 / R^{6}$ power law and is on the order of $1 \mathrm{MHz}$ at $10 \mu \mathrm{m}$ [S2], making it the dominant energy scale in our system for up to several lattice sites.

The coupling between states $|g\rangle$ and $|r\rangle$ is induced by a two-photon transition, with $\left|6 P_{3 / 2}\right\rangle$ as the intermediate level. We drive the transition between $|g\rangle$ and $\left|6 P_{3 / 2}\right\rangle$ with a blue $420 \mathrm{~nm}$ laser (MOGLabs cateye diode laser CEL002) and the transition between $\left|6 P_{3 / 2}\right\rangle$ and $|r\rangle$ with an IR $1013 \mathrm{~nm}$ laser injecting a tapered amplifier (MOGLabs CEL002 and MOA002). The detuning $\delta$ of the blue laser from the $|g\rangle \leftrightarrow\left|6 P_{3 / 2}\right\rangle$ transition is chosen to be much larger than the single-photon Rabi frequencies (typically $\delta \sim 2 \pi \times 560 \mathrm{MHz} \gg \Omega_{B}, \Omega_{R} \sim$ $2 \pi \times 60,36 \mathrm{MHz}$, where $\Omega_{B}$ and $\Omega_{R}$ are the single-photon Rabi frequencies for the blue and red lasers, respectively), such that the dynamics can be safely reduced to a twolevel transition $|g\rangle \leftrightarrow|r\rangle$ driven by an effective Rabi frequency $\Omega=\Omega_{B} \Omega_{R} /(2 \delta) \sim 2 \pi \times 2 \mathrm{MHz}$.

The blue and IR beams are applied counterpropagating to one another along the axis of the array. An external magnetic field is additionally applied, and the beams are circularly polarized such that blue laser drives the $\sigma^{-}$transition between $|g\rangle$ and $|e\rangle=$ $\left|6 P_{3 / 2}, F=3, m_{F}=-3\right\rangle$, while the red laser drives the $\sigma^{+}$transition between $|e\rangle$ and $|r\rangle$. Such a stretched configuration minimizes the probability to excite unwanted states such as $\left|71 S_{1 / 2}, m_{J}=+1 / 2\right\rangle$. The two beams are focused to waists of $20 \mu \mathrm{m}$ (blue) and $30 \mu \mathrm{m}$ (IR) at the position of the atoms, in order to get high intensity while still being able to homogeneously couple all atoms in the array (see section 2.2).

The Rydberg lasers interact with the atoms during one experimental cycle for a few $\mu$ s. In order to maintain laser coherence, the linewidth must be significantly smaller than a few tens of $\mathrm{kHz}$. To achieve this, we use a fast Pound-Drever-Hall scheme to lock our Rydberg lasers to an ultra-low-expansion reference cavity (ATF6010-4 from Stable Laser Systems, with a finesse of 2000 at $420 \mathrm{~nm}$ and $\sim 10000$ at $1013 \mathrm{~nm})$. The optical setup used for this purpose is sketched on Fig. S1b. A fraction of the beam from the blue laser first goes through a phase modulator (Newport 4005) driven by a $18 \mathrm{MHz}$ sinusoidal signal, before being coupled to a longitudinal mode of the reference cavity. The reflected beam from the cavity is sent on a fast photodetector (Thorlabs PDA8A), whose signal is demodulated and low-pass filtered to create an error signal which is fed into a high-bandwidth servo box (Vescent D2-125). The feedback signal from the servo box is applied to the current of the laser diode using a dedicated fast input port on the laser headboard. The measured overall bandwidth of the lock is on the order of $1 \mathrm{MHz}$. The other part of the blue laser beam goes through an acousto-optic modulator (IntraAction ATM1002DA23), whose first diffraction order is used to excite atoms, providing frequency and amplitude control for the Rydberg pulses.

A similar scheme is implemented for the $1013 \mathrm{~nm}$ laser, with the notable difference that the beam used for the frequency lock first goes through a highbandwidth $(>5 \mathrm{GHz})$ fiber-based electro-optic modulator (EOM, EOSpace PM-0S5-05-PFA-PFA-1010/1030). Rather than the carrier, we use a first-order sideband from the EOM for the lock, which makes it possible to 


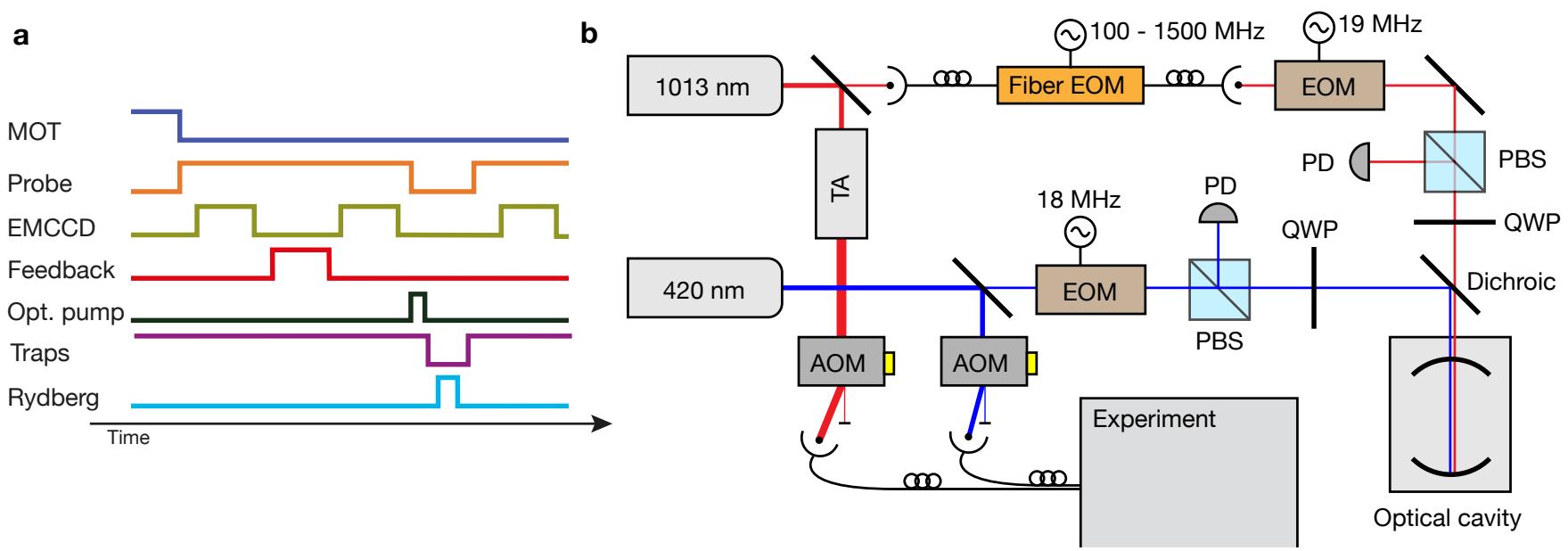

FIG. S1: Experimental sequence and Rydberg laser setup. a, The tweezer array is initially loaded from a MOT. A single-site resolved fluorescence image taken with an electron-multiplied-CCD camera (EMCCD) is used to identify the loaded traps. Using this information, a feedback protocol rearranges the loaded atoms into a preprogrammed configuration, which is verified by the second EMCCD image. After that, all atoms are optically pumped into the $\left|F=2, m_{F}=-2\right\rangle$ state, the tweezers are turned off, and the Rydberg lasers are pulsed. After the traps are turned back on, a third EMCCD image is taken to detect Rydberg excitations with single-site resolution. b, Schematic representation of the Rydberg laser setup, which is used to stabilize two external cavity diode lasers to a reference optical cavity with a fast Pound-Drever-Hall lock. Key: TA=Tapered amplifier, $\mathrm{AOM}=$ Acousto-optic modulator, $\mathrm{EOM}=$ Electo-optic modulator, $\mathrm{PD}=$ Photodetector, $\mathrm{PBS}=\mathrm{Polarizing}$ beam splitter, QWP = Quarter wave plate.

tune the frequency of the red laser over a full free-spectral range of the reference cavity $(1.5 \mathrm{GHz})$ by tuning the driving frequency of the high-bandwidth EOM. Following [S3] and [S4, we estimate that the contribution to the laser linewidth of the noise within the servo loop relative to the cavity is less than $500 \mathrm{~Hz}$ (see section 2.2 for further discussions on laser frequency noise).

\subsection{Detection fidelity}

Each atom is identified as being in $|g\rangle$ (or $|r\rangle$ ) at the end of the Rydberg pulse by whether it is (or is not) present in the third fluorescence image. Detection infidelity arises from accidental loss of atoms in $|g\rangle$ or accidental recapture of atoms in $|r\rangle$. For an atom in state $|g\rangle$, its finite temperature $(\sim 12 \mu \mathrm{K})$ leads to a small probability of not being recaptured after a 'trap off' time of a few microseconds. This results in ground state detection fidelities of $97-99 \%$. In particular, for the 7-atom data shown in Figure 3 in the main text and the 51-atom data shown in Figure 4 and 5, we measured ground state detection fidelities of $98 \%$ and $99 \%$, respectively.

For an atom in state $|r\rangle$, the optical tweezer yields an anti-trapping potential, but there is a finite probability that the atom will decay back to the ground state and be recaptured by the tweezer before it can escape the trapping region. We quantify this probability by measuring Rabi oscillations between $|g\rangle$ and $|r\rangle$ (Fig $\overline{\mathrm{S} 2}$ ) and extracting the maximum amplitude of the oscillation signal. After accounting for the loss of ground state atoms as an offset to the signal, we obtain a typical effective detection fidelity of $93 \%$ for the $\left|71 S_{1 / 2}\right\rangle$ Rydberg state. Furthermore, we observe a reduced detection fidelity at lowerlying Rydberg states, which is consistent with the dependence of the Rydberg lifetime on the principal quantum number [S5].

\section{ADIABATIC PULSES}

\subsection{Pulse optimization}

In order to prepare the ordered phases, we use frequency chirped pulses by varying the two-photon detuning $\Delta$ across the bare $|g\rangle \leftrightarrow|r\rangle$ resonance, corresponding to $\Delta=0$. To perform these sweeps, we drive a high-modulation-bandwidth voltage-controlled oscillator (VCO, Mini-Circuits ZX95-850W-S+) according to either cubic or tangent functional forms:

$$
\begin{gathered}
V(t)_{\text {cubic }}=a\left(t-t_{0}\right)^{3}+b\left(t-t_{0}\right)+\left.c\right|_{\Delta_{\min } \leq \Delta \leq \Delta_{\max }} \\
V(t)_{\text {tangent }}=a \tan \left(b\left(t-t_{0}\right)\right)+\left.c\right|_{\Delta_{\min } \leq \Delta \leq \Delta_{\max }}
\end{gathered}
$$

with programmable parameters $a, b, c$. The output from this VCO is mixed (Mini-Circuits ZFM-2-S+) with a $750 \mathrm{MHz}$ source to generate the difference frequency, which is used to drive the AOM in the $420 \mathrm{~nm}$ light path. The detuning $\Delta$ is set to truncate at minimum 
and maximum values $\Delta_{\min }$ and $\Delta_{\max }$, respectively. The tangent adiabatic sweep has been used for datasets with 51 atoms shown on Figures 4 and 5 of the main text due to improved performance, whereas the cubic form has been used for all smaller system sizes and for the data on crystal dynamics shown on Figure 6 of the main text.

At the end of the sweep, the number of domain walls in the crystal provides a metric for the quality of the crystal preparation. All parameters in (3) or (4) are iteratively optimized as to minimize the domain wall number, i.e. maximize the crystal preparation fidelity. The optimization starts with the offset $c$, followed by the $b$ parameter, then the maximum and minimum detunings $\Delta_{\min / \max }$, and finally the $a$ parameter. Repeated optimization of these parameters often leads to better crystal preparation fidelities.

After passing through the AOM, the $420 \mathrm{~nm}$ light is coupled into a fiber. The coupling is optimized for the VCO frequency at which the light is resonant with the $|g\rangle$ to $|r\rangle$ transition $\left(f_{\text {opt }}\right)$, and decreases as the VCO frequency deviates from $f_{\text {opt }}$. The power throughout all frequency sweeps is $\geq 75 \%$ of the power at $f_{\text {opt }}$.

\subsection{Limitations}

When sweeping into the crystalline phase, the control parameter $\Delta(t)$ must be varied slowly enough that the adiabaticity criterion is sufficiently met. However, for long pulses, additional technical errors may become limiting. Here we summarize some key limitations:

- State preparation fidelity: For all analyzed data, we preselect on defect-free atom arrays. Preparation fidelity is therefore given by the probability that each atom in the array is still present for the Rydberg pulse, and that it is prepared in the correct magnetic sublevel: $\left|5 S_{1 / 2}, F=2, m_{F}=-2\right\rangle$. Including both factors, we estimate that atoms are present and in the correct magnetic sublevel with fidelity $f>98 \%$. For experiments with 51 atoms, this leads to $\lesssim 1$ atom incorrectly prepared.

- Spontaneous emission: The $71 S$ Rydberg state has an estimated lifetime of $150 \mu$ s (including blackbody radiation at $300 \mathrm{~K}$ ) S5]. Additionally, for the typical intermediate detuning $\Delta \approx 2 \pi \times 560 \mathrm{MHz}$ and single photon IR and blue Rabi frequencies of $\left(\Omega_{R}, \Omega_{B}\right) \approx 2 \pi \times(36,60) \mathrm{MHz}$, spontaneous emission from the intermediate state occurs on a time scale of $40 \mu$ s for the ground state, and introduces a combined effective lifetime of $50 \mu$ s for the Rydberg state. This leads to an average scattering rate of $2 \pi \times 3.6 \mathrm{kHz}$.

- Rabi frequency homogeneity: We aim to align our beams to globally address all trapped atoms with a uniform Rabi frequency $\left|\Omega_{i}\right|=\Omega$. Experimentally, we achieve homogeneity up to differences $\lesssim 3 \%$ (Fig. S2b).

- Intensity fluctuations: Primarily because of pointing instability, the global Rabi frequency fluctuates by small amounts from shot to shot of the experiment. In order to reduce slow drifts of the beams, we use a 1:1.25 telescope to image on a camera their position on the plane of the atoms and feedback to stabilize their position to a target every 500 repetitions ( $\sim 2$ minutes).

- Rydberg lasers frequency noise: The fast $(\sim \mathrm{MHz})$ fluctuations of the error signal when the Rydberg lasers are locked correspond to random detuning fluctuations with $\simeq 15 \mathrm{kHz}$ RMS amplitude, which is much smaller than the Rabi frequency itself and is expected not to play a role in the experiments described in this paper. More generally, the good coherence properties of our Rydberg lasers over our typical experimental times have been confirmed by spin echo measurements between $|g\rangle$ and $|r\rangle$ for non-interacting atoms, which showed no visible decay of coherence over $5 \mu$ s (Fig. S2 ).

- Finite atomic temperature: Our finite atomic temperature of $\sim 12 \mu \mathrm{K}$ introduces both random Doppler shifts (of order $2 \pi \times 50 \mathrm{kHz}$ ), as well as fluctuations in the atomic positions $(\sim 120 \mathrm{~nm}$ radially, $\sim 600 \mathrm{~nm}$ longitudinally) for each atom in each cycle of the experiment. The Doppler shift is very small in magnitude compared to the single atom Rabi frequency $\Omega$. The position fluctuations can introduce noticeable fluctuations in the interaction energy between a pair of atoms from shot to shot. As an example, at our chosen lattice spacing of $5.9 \mu \mathrm{m}$, we calculate an interaction energy of $2 \pi \times 24 \mathrm{MHz}$. However, if the distance fluctuates by values on the order of $\sqrt{2} \times 120 \mathrm{~nm} \approx 170 \mathrm{~nm}$, then the actual interaction energy can range from $21 \mathrm{MHz}$ to $29 \mathrm{MHz}$. The longitudinal position fluctuations add in quadrature, so they contribute less to fluctuations in distance.

- Electric and magnetic fields: We have observed that the Rydberg resonance can drift over time, especially for states with high principal quantum number $n$, which we attribute to uncontrolled fluctuations in the electric field. We can reduce these fluctuations by shining UV light at $365 \mathrm{~nm}$ on the glass cell in between experimental sequences and during the MOT loading period, which stabilizes the charge environment on the glass cell surface. While the fluctuations for states $n \geq 100$ are still significant, they become negligible $(<100 \mathrm{kHz})$ for our chosen state $n=71$. 

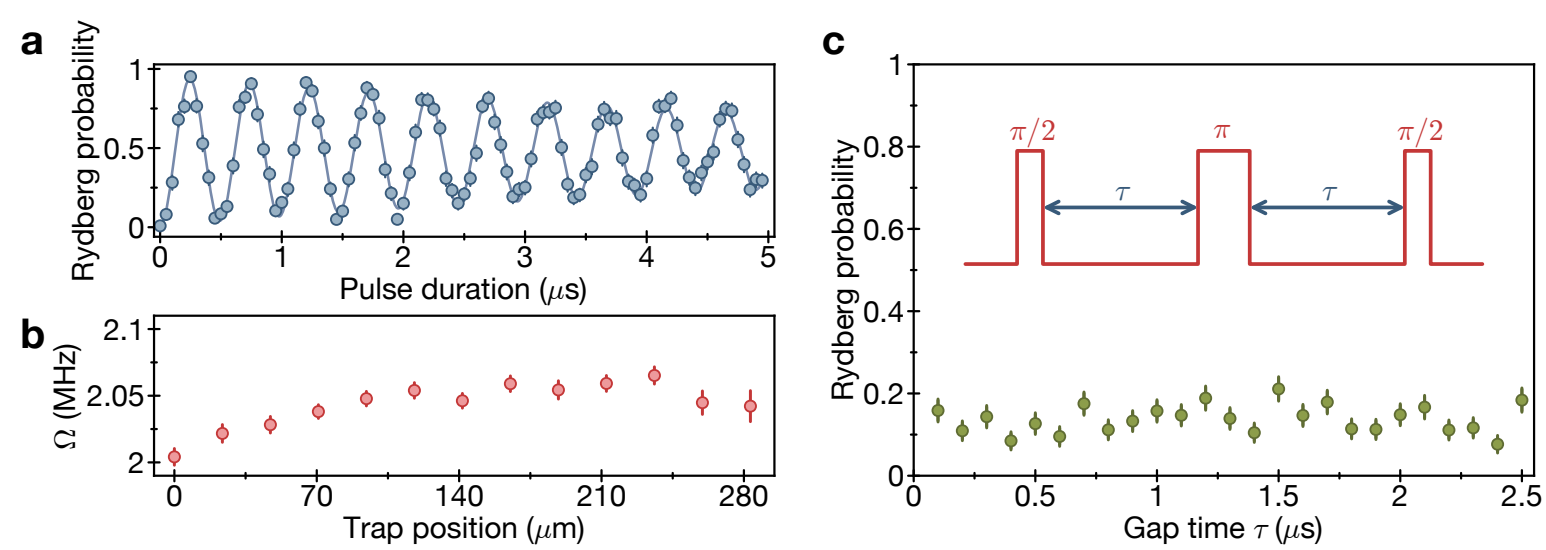

FIG. S2: Typical Rabi oscillation, homogeneity and coherence for non-interacting atoms $\left(\mathrm{a}=24 \mu \mathrm{m}, \Omega \gg V_{i, i+1} \simeq\right.$ $5 \mathrm{kHz}$ ). a, Rabi oscillations. We observe a typical decay time of $\sim 6 \mu \mathrm{s}$, which is mainly limited by intensity fluctuations from shot to shot. $\mathbf{b}$, The fitted Rabi frequency for each atom across the array (spatial extent $\sim 300 \mu \mathrm{m}$ ) is homogeneous to within $<3 \%$. Error bars denote $68 \%$ confidence intervals. c, Measurement of the population in the Rydberg state after a spin echo pulse sequence drawn above. We find no decay of coherence over typical measurement periods of several microseconds, thereby ruling out fast sources of decoherence.

The energy shifts of the initial state $|g\rangle$ and final state $|r\rangle$ with magnetic fields are identical. Differential shifts of the intermediate state are very small compared to the detunings of the two laser beams from the $6 P_{3 / 2}$ state. Therefore, we do not expect magnetic fields to play any significant role in fluctuations between experimental runs.

\section{CORRECTING FOR FINITE DETECTION FIDELITY}

The number of domain walls is a metric for the quality of preparing the desired crystal state. Boundary conditions make it favorable to excite the atoms at the edges. Therefore, we define a domain wall as any instance where two neighboring atoms are found in the same state or an atom at the edge of the array is found in state $|g\rangle$. In systems composed of an odd number of particles, this definition sets the parity of domain walls to be even.

The appearance of domain walls can arise from nonadiabaticity across the phase transition, as well as scattering from the intermediate $6 P$ state, imperfect optical pumping, atom loss, and other processes (see section 2.2). However, the observed number of domain walls is artificially increased owing to detection infidelity; any atom within a crystal domain that is misidentified increases the number of measured domain walls by two. For this reason, we use a maximum-likelihood routine to estimate the parent distribution, which is the distribution of domain walls in the prepared state that best predicts the measured distribution. We use two methods to correct for detection infidelity, depending on whether we are interested only in the probability to generate the many- body ground state, or in the full probability distribution of the number of domain walls.

\subsection{Many-body ground state preparation}

Having prepared the many-body ground state, the probability to correctly observe it depends on the measurement fidelity for atoms in the electronic ground state $f_{g}$, the measurement fidelity for atoms in the Rydberg state $f_{r}$, and the size of the system $N$. Assuming a perfect crystal state in the $Z_{2}$ phase, the total number of atoms in the Rydberg state is $n_{r}=(N+1) / 2$, while the number of atoms in the ground state is $n_{g}=(N-1) / 2$. The probability to measure the perfect state is then $p_{m}=f_{r}^{n_{r}} \times f_{g}^{n_{g}}$. Therefore, if we observe the ground state with probability $p_{\exp }$, the probability of actually preparing this state is inferred to be $p_{\exp } / p_{m}$. The blue data points in Fig. $4 \mathrm{a}$ in the main text are calculated this way.

\subsection{Maximum likelihood state reconstruction}

In order to correct for detection fidelity in the entire distribution of domain walls, we use a maximumlikelihood protocol. For this purpose, we assume that the density of domain walls is low, such that the probability of preparing two overlapping domain walls, meaning three consecutive atoms in the same state, is negligibly small. Under this assumption, misidentifying an atom within a domain wall shifts its location, but does not change the total number. However, misidentification of an atom within a crystal domain increases the 


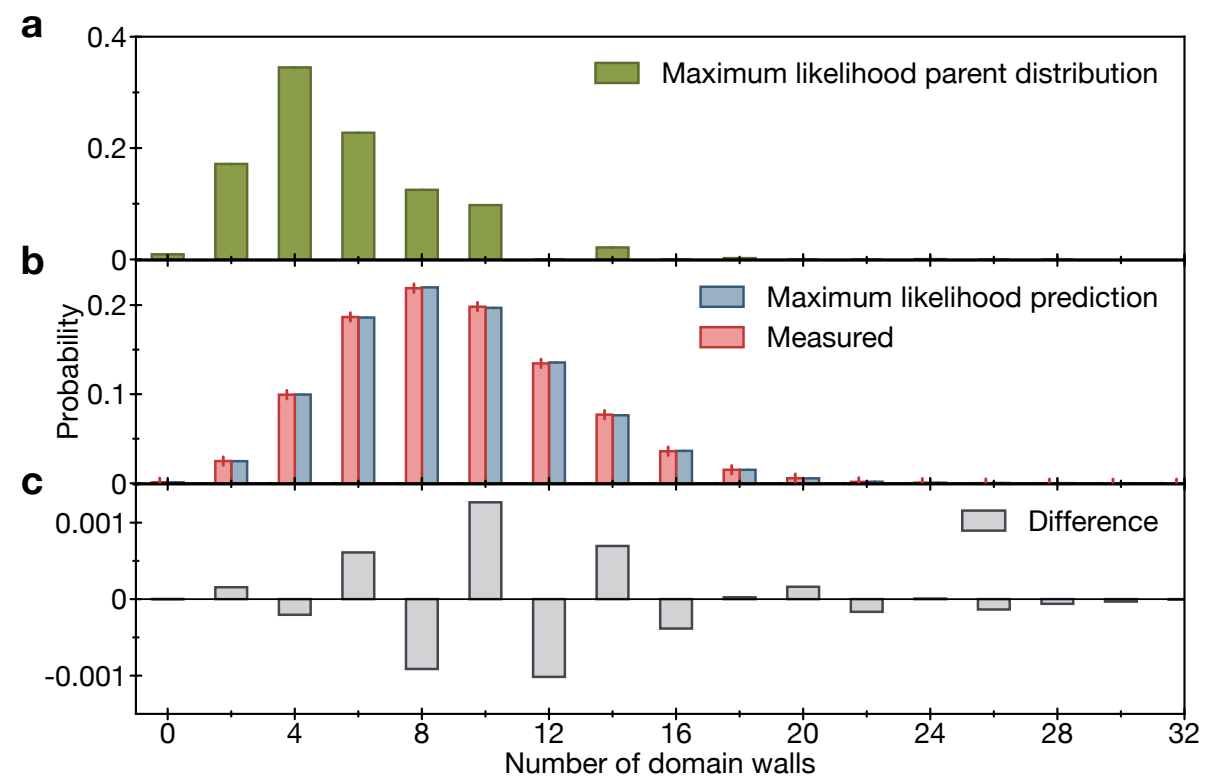

FIG. S3: State reconstruction. a, Reconstructed parent distribution. b, Comparison of measured domain wall distribution (red) and predicted observation given the parent distribution in a (blue). c, Difference between the two distributions in $\mathbf{b}$.

number of domain walls by two. For any prepared state with a number of domain walls $n_{i}$, we can calculate the probability to measure $n_{f}$ domain walls, $p\left(n_{f} \mid n_{i}\right)$. We can construct a matrix $M$, which transforms an initial probability distribution in number of domain walls, $\vec{W}_{i}=\left(p\left(n_{i}=0\right), p\left(n_{i}=2\right), \ldots\right)$, into the expected observed distribution $\vec{W}_{f}=M \vec{W}_{i}$, where $M_{k l}=p\left(n_{f}=\right.$ $\left.k \mid n_{i}=l\right)$. Given an experimentally observed distribution of domain walls, $\vec{W}_{o}$, and a test initial distribution $\vec{W}_{i}^{\prime}$, we can calculate the difference vector between them $\overrightarrow{D^{\prime}}=\vec{W}_{o}-\vec{W}_{f}^{\prime}=\vec{W}_{o}-M \vec{W}_{i}^{\prime}$.

Using $\vec{D}^{\prime}$ and the confidence intervals of the measured data $(\vec{\sigma})$, we define a cost function

$$
C\left(\vec{W}_{o}, \vec{W}_{i}^{\prime}\right)=\sum_{k}\left(\frac{D_{k}^{\prime}}{\sigma_{k}}\right)^{2},
$$

where $\vec{\sigma}$ represents the $68 \%$ confidence intervals obtained via an approximate parametric bootstrap method [S6], and the sum is taken over the elements of the vectors. We can find the most likely parent distribution, $\vec{W}_{i}$, by minimizing the cost function over the different possible $\vec{W}_{i}^{\prime}$, under the constraint that that every element is between 0 and 1 , and the sum of the elements is 1 . For this purpose, we use a Sequential Least Square Programming routine. To reduce biases, we use a random vector as a starting point of the minimization procedure. We checked that repeating the procedure several times with different initial vectors converged to the same parent distribution, and that the distribution of domain walls predicted by this parent distribution was in excellent agreement with the measured distribution. The result of such a procedure on the dataset used for Fig. 5c of the main text is shown in Fig. S3.

\section{COMPARISON WITH A CLASSICAL THERMAL STATE}

To gain some insight into the states obtained from our preparation protocol (Fig. 3a in the main text), we provide a quantitative comparison between experimentally measured quantities and those computed from a thermal ensemble. In particular, we note that deep in the ordered phase $\Delta / \Omega \gg 1$, the coherent coupling of the ground state to the Rydberg state can be neglected owing to strong energetic suppression, and that the effective Hamiltonian becomes diagonal in the measurement basis. This allows us to calculate all properties of a thermal state even for systems of 51 atoms by explicitly computing the partition function via the transfer matrix method [S7]. Also, we may consider the interactions only up to next-nearest neighbors as the coupling strengths for longer distances are weak compared to the maximum timescale accessible in our experiments. To this end, we consider the Hamiltonian $\mathcal{H}_{\mathrm{cl}}=-\Delta \sum_{i=1}^{N} n_{i}+\sum_{i=1}^{N-1} V_{1} n_{i} n_{i+1}+\sum_{i=1}^{N-2} V_{2} n_{i} n_{i+2}$.

The eigenstates of this Hamiltonian are simply $2^{N}$ classical configurations, where each atom is in either $|g\rangle$ or $|r\rangle$. We label these configurations by a length- $N$ vector $\vec{i}=\left(i_{1}, i_{2}, \ldots, i_{N}\right)\left(i_{n} \in\{g, r\}\right)$, and denote their energy by $E_{\vec{i}}$. In a thermal ensemble $\rho=\exp \left(-\beta \mathcal{H}_{\mathrm{cl}}\right) / Z$ with $Z \equiv \operatorname{tr}\left[\exp \left(-\beta \mathcal{H}_{\mathrm{cl}}\right)\right]$ and inverse temperature $\beta$, the 

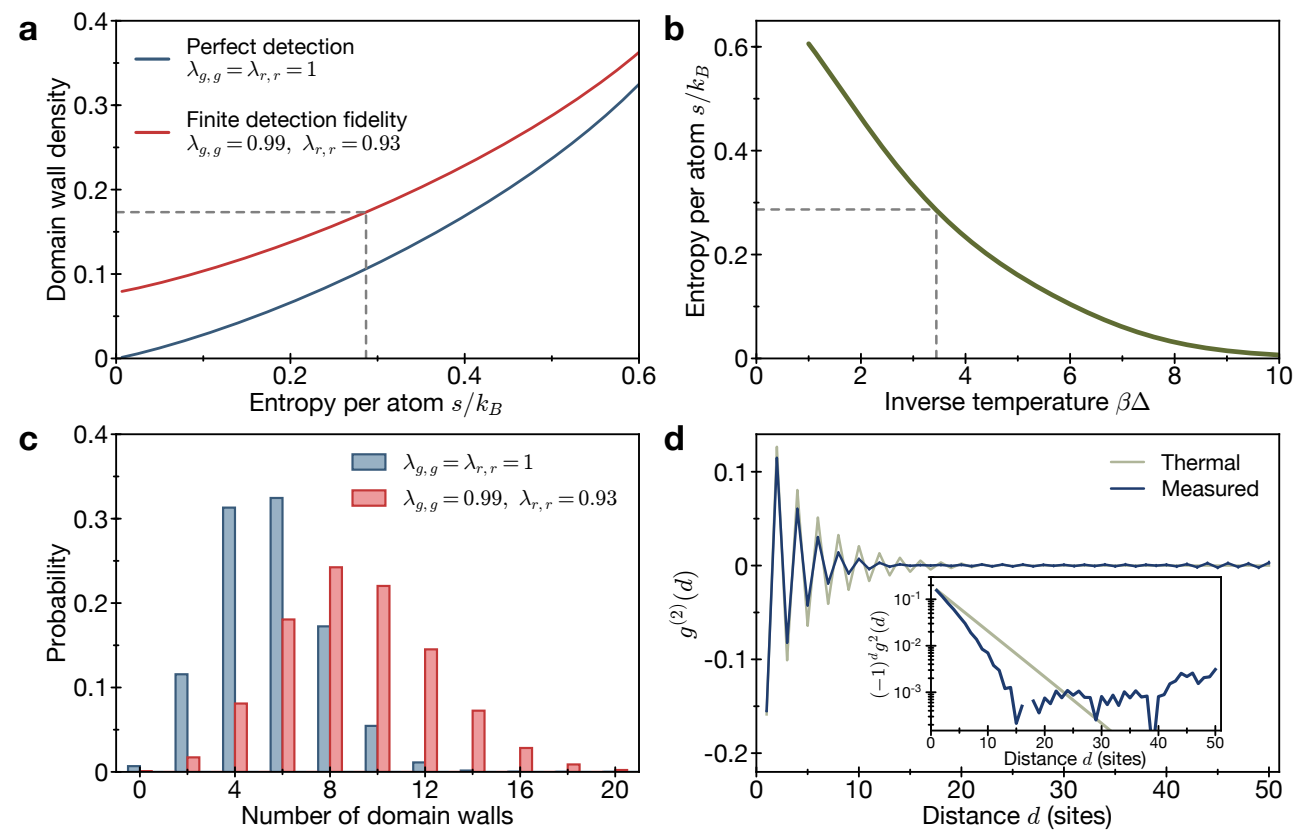

FIG. S4: Comparison to thermal state. a, Domain wall density for thermal states at different entropy per atom. The lower line corresponds to the actual number of domain walls in a system of the corresponding temperature, the upper line gives the domain wall density one would measure at this temperature, given the finite detection fidelity. The horizontal dashed line denotes the experimentally measured domain wall density, from which we can infer a corresponding entropy per atom and equivalently, temperature, in a thermal ensemble. b, Entropy per atoms for a thermal state at given inverse temperature $\beta=1 /\left(k_{B} T\right)$ in a 51-atom array. c, Expected distribution of the number of domain walls for the thermal ensemble at $\beta=3.44 / \Delta$, with (red) and without (blue) taking into account finite detection fidelity. d, Experimentally measured correlation function $g^{(2)}(d)$ and correlation function corresponding to a thermal ensemble at $\beta=3.44 / \Delta$. The inset shows the rectified correlation function on a logarithmic scale, indicating that the measured correlation function decays exponentially, but with a different correlation length than one obtains from a thermal state with the measured number of domain walls.

probability to find a particular configuration $\vec{i}$ is given by $p_{\vec{i}}=\exp \left(-\beta E_{\vec{i}}\right) / Z$. Since $E_{\vec{i}}$ can be written as a sum of local terms involving only interactions up to a range 2 , the partition sum can be evaluated using a standard transfer matrix of size $4 \times 4$. Moreover, using this approach, we can evaluate all measurable quantities for the thermal ensemble such as the average number of domain walls $\langle D\rangle=\operatorname{tr}\{D \rho\}$, where $D$ is an operator counting the number of domain walls, i.e. $D=\sum_{i=1}^{N-1}\left(n_{i} n_{i+1}+\right.$ $\left.\left(1-n_{i}\right)\left(1-n_{i+1}\right)\right)+\left(1-n_{1}\right)+\left(1-n_{N}\right)$, the correlation function $g^{(2)}(d)=1 /(N-d) \sum_{i=1}^{N-d} g_{i, i+d}^{(2)}$, and even the full counting statistics for the domain wall distribution in the state $\rho$. In particular, the probability to measure exactly $n$ domain walls $p_{n}=\operatorname{tr}\left\{P_{n} \rho\right\}$ can be computed from a Fourier transform of the Kronecker delta function $P_{n} \equiv \delta_{D, n}=\frac{1}{N+2} \sum_{k=0}^{N+1} \exp \left[i \frac{2 \pi}{N+2} k(n-D)\right]$ with $n=0,1,2, \ldots N+1$.

One can directly include the effect of imperfect detections in this formalism. To that end, we denote the expectation value of an observable $O$ as

$$
\left\langle\langle O\rangle=\sum_{\vec{i}, \vec{j}} O_{\vec{i}} \Lambda_{\vec{i}, \vec{j}} p_{\vec{j}}\right.
$$

where $O_{\vec{i}}$ is the value of the observable in state $\vec{i}$, and $\Lambda_{\vec{i}, \vec{j}}$ is the probability to detect state $\vec{i}$ when the system is in state $\vec{j}$, accounting for finite detection fidelity. Assuming detection errors occur independently from one another, we have $\Lambda_{\vec{i}, \vec{j}}=\prod_{n} \lambda_{i_{n}, j_{n}}$ where $\lambda_{g, g}=f_{g}$ is the probability to correctly detect an atom in the ground state, $\lambda_{r, r}=f_{r}$ is the probability to correctly detect an atom in the Rydberg state, and $\lambda_{r, g}=1-\lambda_{g, g}$, and $\lambda_{g, r}=1-\lambda_{r, r}$. Equation (6) can be evaluated using a $16 \times 16$ transfer matrix for any observables of interest.

In order to get a quantitative comparison with our experiments, we determine the inverse temperature $\beta$ in such a way that the average number of domain walls $\langle\langle D\rangle\rangle$, including the effect of imperfect detections, matches the experimentally determined value, i.e. $\langle\langle D\rangle\rangle=9.01(2)$. For $\Delta=2 \pi \times 14 \mathrm{MHz}, V_{1}=2 \pi \times 24 \mathrm{MHz}$ and $V_{2}=2 \pi \times 0.38 \mathrm{MHz}$ this leads to $\beta=3.44(1) / \Delta$ or equivalently to the entropy per atom of $s / k_{B}=0.286(1)$ (Fig. S4, $\mathrm{b}$ ). Since $\beta$ characterizes the thermal state completely, we can extract the corresponding domain wall distribution (Fig. S4 ) and the correlation function (Fig. $\mathrm{S} 4 \mathrm{~d}$ ) as described above. We find that the correlation length in the corresponding thermal state is 
$\xi_{\text {th }}=4.48(3)$, which is significantly longer than the measured correlation length $\xi=3.03(6)$, from which we deduce that the experimentally prepared state is not thermal.

\section{DYNAMICS AFTER SUDDEN QUENCH}

\subsection{Matrix Product State Ansatz}

To understand the dynamics of the $Z_{2}$ Rydberg crystal following the quench of the detuning to $\Delta=0$, we first consider a simplified model, where interactions beyond nearest neighbor are neglected. In addition, we replace the nearest neighbor interactions with hard constraints that two neighboring atoms cannot be excited at the same time; such an approximation is well controlled in the limit of $V_{i, i+1} \gg \Omega$, as in the case of our experiments, for a time exponentially long in $V_{i, i+1} / \Omega$ [S8]. In this limit, the Hamiltonian can then be approximated by

$$
\mathcal{H}_{\mathrm{c}}=\sum_{i} P_{g}^{i-1}\left(\frac{\Omega}{2} \sigma_{x}^{i}-\Delta P_{r}^{i}\right) P_{g}^{i+1},
$$

where $P_{g}^{i}=\left|g_{i}\right\rangle\left\langle g_{i}\left|, P_{r}^{i}=\right| r_{i}\right\rangle\left\langle r_{i}\right|$. We identify $P_{g}^{i=0}=$ $P_{g}^{i=N+1}=1$ at the boundaries. Within this approximation, the relevant Hilbert space consists only of states with no neighboring atoms in the Rydberg state, i.e. $P_{r}^{i} P_{r}^{i+1}=0$. The dimension of this constrained Hilbert space is still exponentially large and grows as $\sim \phi^{N}$, where $\phi=1.618 \ldots$ is the golden ratio.

In the simplest approximation, one can treat the array of atoms as a collection of independent dimers, $|\Psi(t)\rangle=\bigotimes_{i}|\phi(t)\rangle_{2 i-1,2 i}$, where for each pair of atoms only three states are allowed due to the blockade constraint, $|r, g\rangle,|g, g\rangle$ and $|g, r\rangle$. The dynamics of each pair with initial state $|\phi(0)\rangle=|r, g\rangle$ is then given by $|\phi(t)\rangle=\frac{1}{2}(1+\cos (\Omega t / \sqrt{2}))|r, g\rangle+\frac{i}{\sqrt{2}} \sin (\Omega t / \sqrt{2})|g, g\rangle+$ $\frac{1}{2}(1-\cos (\Omega t / \sqrt{2}))|g, r\rangle$. This dimer model predicts that each atom flips its state with respect to its initial configuration after a time $\tau=\sqrt{2} \pi / \Omega$. The corresponding oscillations between two complementary crystal configurations are thus a factor $\sqrt{2}$ slower than an independent spin model would predict, which is qualitatively consistent with the experimental observations. We note that this dimerized ansatz does not satisfy the constraint $P_{r}^{i} P_{r}^{i+1}=0$ between two neighboring dimers, which is an artifact originating from the artificial partitioning of the array into non-interacting dimers.

To go beyond this approximation, we consider an ansatz for the many-body wavefunction that treats each atom on an equal footing. The simplest such wavefunction that also allows for non-trivial entanglement between the atoms can be written as a matrix product state (MPS) with bond dimension 2. In particular we consider a manifold of states of the form $\left|\Psi\left(\left\{\theta_{n}\right\}\right)\right\rangle=$

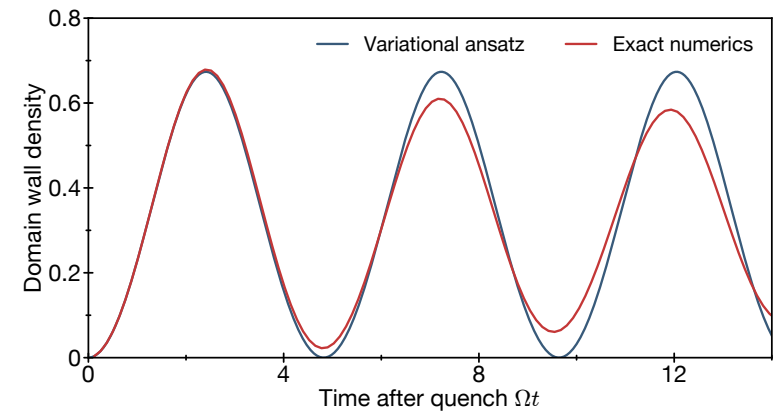

FIG. S5: Oscillations of domain wall density: Using a variational matrix product state ansatz. Dynamics of the domain wall density in the bulk of the array under the constrained Hamiltonian $\mathcal{H}_{\mathrm{c}}$ at $\Delta=0$. The blue line shows the evolution of the domain wall density obtained from integrating the variational equation of motion eq. (9) with initial conditions $\theta_{a}=\pi / 2, \theta_{b}=0$, i.e. the crystalline initial state. The red line shows the exact dynamics of the domain wall density at the center of a system of 25 atoms initially in the crystalline state under the constrained Hamiltonian $\mathcal{H}_{\mathrm{c}}$.

$\sum_{\left\{i_{n}\right\}} v_{L} A\left(\theta_{1}\right)^{i_{1}} A\left(\theta_{2}\right)^{i_{2}} \cdots A\left(\theta_{N}\right)^{i_{N}} v_{R}\left|i_{1}, i_{2}, \ldots, i_{N}\right\rangle$ with matrices

$$
A\left(\theta_{n}\right)^{g}=\left(\begin{array}{cc}
\cos \left(\theta_{n}\right) & 0 \\
1 & 0
\end{array}\right) \quad A\left(\theta_{n}\right)^{r}=\left(\begin{array}{cc}
0 & i \sin \left(\theta_{n}\right) \\
0 & 0
\end{array}\right),
$$

and boundary vectors $v_{L}=\left(\begin{array}{ll}1 & 1\end{array}\right)$ and $v_{R}=\left(\begin{array}{ll}1 & 0\end{array}\right)^{\top}$. Here, the indices $i_{n} \in\{g, r\}$ enumerate the state of the $n$-th atom. This manifold satisfies the constraint that no two neighboring atoms are simultaneously excited. The many-body state within this subspace is completely specified by the $N$ parameters $\theta_{n} \in[0,2 \pi]$. In particular, it allows to represent the initial crystal state, $\theta_{2 n-1}=\pi / 2$ for atoms on odd sites and $\theta_{2 n}=0$ for atoms on even sites, as well as its inverted version, $\theta_{2 n-1}=0$ for odd and $\theta_{2 n}=\pi / 2$ for even sites, respectively. Using the time-dependent variational principle [S9], we derive equations of motion for the wave function within this manifold. For an infinite system with a staggered initial state $\theta_{n+2}=\theta_{n}$, such as the $Z_{2}$ ordered state, the wave function is at all times described by two parameters $\theta_{a}=\theta_{2 n-1}$ and $\theta_{b}=\theta_{2 n}$ for even and odd sites. The corresponding non-linear, coupled equations of motion read

$$
\begin{aligned}
& \dot{\theta}_{a}=-\frac{1}{2} \sec \left(\theta_{b}\right)\left(\sin \left(\theta_{a}\right) \cos ^{2}\left(\theta_{a}\right) \sin \left(\theta_{b}\right)+\cos ^{2}\left(\theta_{b}\right)\right) \\
& \dot{\theta}_{b}=-\frac{1}{2} \sec \left(\theta_{a}\right)\left(\sin \left(\theta_{b}\right) \cos ^{2}\left(\theta_{b}\right) \sin \left(\theta_{a}\right)+\cos ^{2}\left(\theta_{a}\right)\right) .
\end{aligned}
$$

A numerical solution of these variational equations for the crystalline initial state predicts a periodic motion with a frequency of $\approx \Omega / 1.51$, where the many-body 


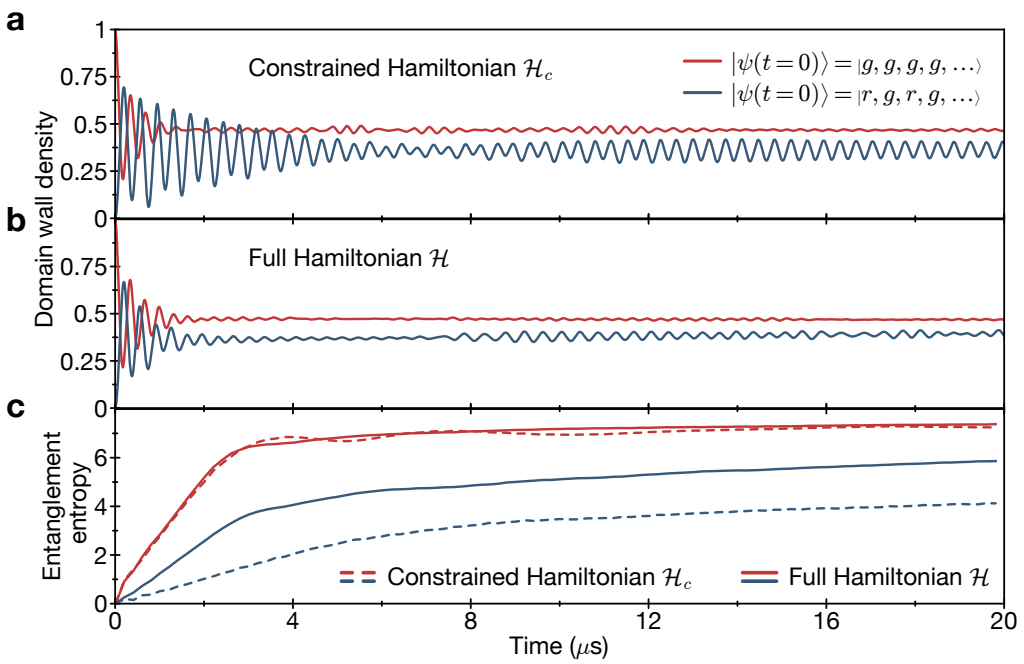

FIG. S6: Decay of oscillations after a quench and entropy growth. a, Dynamics of the domain wall density under the constrained Hamiltonian $\mathcal{H}_{\mathrm{c}}$ for different initial states. The red line shows the domain wall density for a system of 25 atoms initially prepared in the electronic ground state. In this case, the domain wall density quickly relaxes to a steady value corresponding to thermalization. In contrast, the blue line shows the dynamics if the system is initialized in the $Z_{2}$ ordered state. The domain wall density oscillates over several periods and even for very long times does not fully relax to a steady value. b, Same as in a but taking into account the full $1 / R^{6}$ interactions. While the dynamics for an initial state $|g\rangle^{\otimes N}$ is very similar to the one obtained in the constrained case, for the crystalline initial state the decay of the oscillations is faster than in the constrained model. c, Growth of entanglement entropy in a bipartite splitting of the 25 atom array for the different cases displayed in $\mathbf{a}$ and $\mathbf{b}$. The entropy is defined as the von Neumann Entropy of the reduced state of the first 13 atoms of the array. The dashed lines correspond to dynamics under the constrained Hamiltonian, neglecting the $1 / R^{6}$ tail, while the solid lines take the full interactions into account. Red lines correspond to the initial state $|g\rangle^{\otimes N}$, while blue lines correspond to crystalline initial states. In all panes we chose $\Omega=2 \pi \times 2 \mathrm{MHz}$, and where applicable, interaction parameters such that the nearest neighbor interaction evaluates to $V_{i, i+1}=2 \pi \times 25.6 \mathrm{MHz}$.

wavefunction oscillate between two staggered configurations.

\subsection{Decay of the oscillations and growth of entanglement}

In order to obtain more insight into the dynamics of our system beyond these variational models, we use exact numerical simulations to integrate the many-body Schrödinger equation. In particular, we focus on the decay of oscillations and the growth of entanglement entropy in our system. Due to the exponentially growing Hilbert space, this method is limited to relatively small system sizes. We make use of the constrained size of the Hilbert space (blockade of nearest neighboring excitations of Rydberg states), and propagate the state vector of up to 25 spins using a Krylov subspace projection method. In Fig. S6 a we show the dynamics of the domain wall density under the time evolution of the constrained Hamiltonian $\mathcal{H}_{c}$ with $\Omega=2 \pi \times 2 \mathrm{MHz}$ and $\Delta=0$. We consider two different initial states: the disordered state where each atom is initially prepared in the ground state $|g\rangle$, and the perfect crystalline state $|r, g, r, g, \ldots\rangle$. We note that in both cases the energy density corresponds to that of an infinite temperature thermal ensemble in the constrained subspace with respect to $\mathcal{H}_{c}$.

For the disordered initial state, the domain wall density quickly relaxes to a steady state value. In contrast, if the system is initialized in the perfect crystalline state, the domain wall density oscillates for long times and decays at a rate much slower than the oscillation period. We confirmed numerically that this initial decay time is independent of the system size. We further note that for every system size accessible in our numerical method, the domain wall density does not relax to a steady value even at very long times, but continues to oscillate with a reduced amplitude. Moreover, while the disordered initial state relaxes to an average domain wall density consistent with a thermal state of infinite temperature corresponding to the energy density of the initial state, this is clearly not the case for the crystalline initial state. This qualitatively distinct behavior for two different initial states is also reflected in the growth of entanglement entropy after the quench, shown in Fig. S6p (dashed lines). While in both cases the entanglement entropy grows initially linearly, the rate of growth is significantly lower for the crystalline initial state. Moreover, unlike the case of disordered initial state where the entanglement entropy quickly saturates to its maximum value (limited by the finite system 


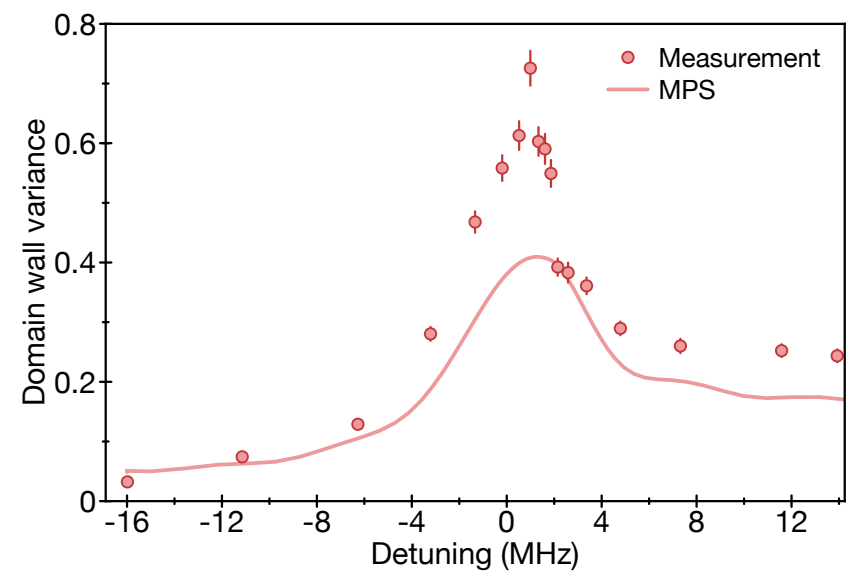

FIG. S7: Variance of the domain wall distribution during $\mathbf{Z}_{2}$ state preparation. Points and error bars represent measured values. The solid red line corresponds to a full numerical simulation of the dynamics using a matrix product state ansatz (see text and Fig. 5 of the main text)

size and the constrained Hilbert space), for the crystalline initial state, the entanglement entropy does not seem to approach the same value.

To understand the influence of the $1 / R^{6}$-decaying interactions, we show the corresponding dynamics and entanglement growth in Fig. $\mathrm{S} 6 \mathrm{p}$ and $\mathrm{c}$ (solid lines). Numerically, we treat the strong nearest neighbor interactions perturbatively - by adiabatic eliminations of simultaneous excitation of neighboring Rydberg states while the weak interactions beyond nearest neighbors are treated exactly. For the disordered initial state, we find that the dynamics of domain wall density and the entanglement growth remain similar to the previous case, where long range interactions are neglected; in this case, the thermalization time is barely affected. In contrast, for the crystalline initial state, the oscillations decay significantly faster once next-to-nearest neighbor interactions are included. We thus attribute the thermalization in this case to interactions beyond the nearest neighbor blockade constraint. From the growth of the entanglement entropy, we see that the crystalline initial state still thermalizes slower than the disordered initial state.

\subsection{Time evolution via matrix product state algorithm}

The numerical data presented in Fig. 5b and Fig. 6b in the main text are obtained by simulating the evolution of the 51 atom array during the sweep across the phase transition as well as the subsequent sudden quench using a matrix product state algorithm with bond dimension $D=256$. We simulate the entire preparation protocol to generate the Rydberg crystal [Fig. 5 b in the main text], and use the resulting state as an initial state for the time evolution after the sudden quench. To this end, we use a time-evolving block decimation (TEBD) algorithm S10, S11, with a Suzuki-Trotter splitting of the Hamiltonian to update the state. The time step used in this Trotterization is $\Omega \Delta t=0.004$. We take into account only nearest neighbor and next-nearest neighbor interactions and neglect small interactions for atoms that are separated by 3 or more sites (as discussed also in Sec. 4). We account for finite detection fidelities that are determined independently, but otherwise do not include any incoherent mechanisms. Remarkably, for local quantities, such as the domain wall density, this fully coherent simulation agrees well with the experimentally measured values. For higher-order correlation functions, such as the variance of the domain wall number, the fully coherent simulation and the experiment agree only qualitatively (Fig. S7). The quantitative difference is likely due to either limitations of the MPS simulations or various incoherent processes present in the experiment.

[S1] M. Endres et al., Science 354, 1024 (2016)

[S2] K. Singer, J. Stanojevic, M. Weidemüller, and R. Côté, J. Phys. B: At. Mol. Opt. Phys. 38, S295 (2005)

[S3] J. L. Hall and M. Zhu, in Laser Manipulation of Atoms and Ions, Proceedings International School of Physics "Enrico Fermi" Vol. CXVIII, 671 (1992)

[S4] R. W. Fox, C. W. Oates and L. W. Hollberg, in Experimental Methods in the Physical Sciences, Vol. 40, 1 (2003)

[S5] I. I. Ryabtsev, D. B. Tretyakov, and V. M. Entin, Phys. Rev. A 79, 052504 (2009)

[S6] J. Glaz and C. P. Sison, J. Stat. Plan. Inference 82, 251 (1999)

[S7] R. J. Baxter, Exactly Solved Models in Statistical Mechanics (2007)

[S8] D. Abanin, W. De Roeck, W. W. Ho, and F. Huveneers, Comm. Math. Phys. 354, 809 (2017)o

[S9] J. Haegeman et al., Phys. Rev. Lett. 107, 070601 (2011)

[S10] G. Vidal, Phys. Rev. Lett. 93, 040502 (2004)

[S11] A. J. Daley, C. Kollath, U. Schollwöck, and G. Vidal, J. Stat. Mech. Theor. Exp. 2004, P04005 (2004) 